

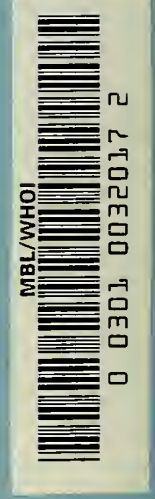




\section{A STUDY OF THE FORCES AND MOMENTS \\ ON A HEAVING SURFACE SHIP}

by

Paul Golovato

September 1957

Report 1074

NS715-102 
TABLE OF CONTENTS

Page

ABSTRACT ……

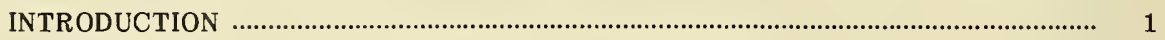

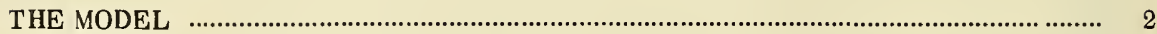

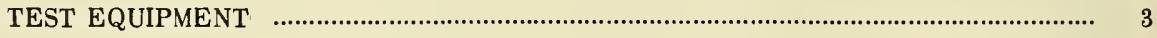

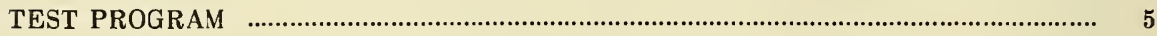

METHOD OF ANALYSIS

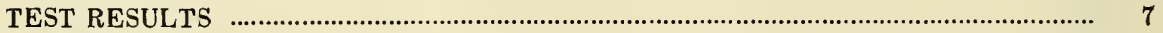

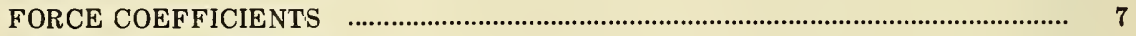

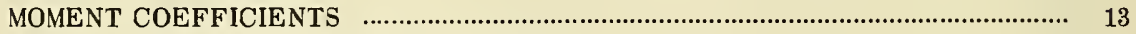

SECOND HARMONICS IN THE LIFT FORCE

DISCUSSION

PREDICTION OF DAMPING-IN-HEAVE ……

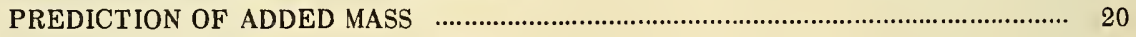

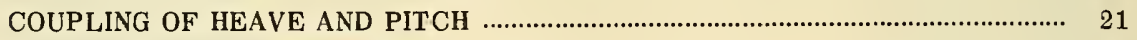

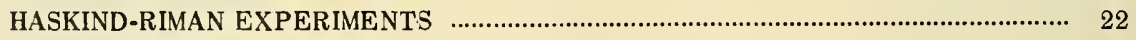

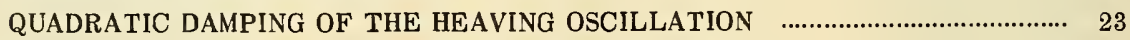

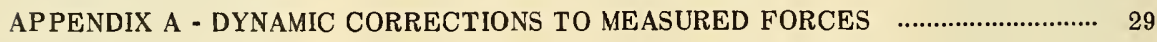

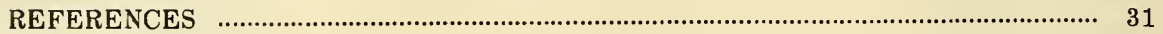




\begin{abstract}
A surface ship model was constrained to perform forced heaving oscillations in still water and the resulting lift forces and pitching moments obtained. The influence of forward speed, frequency and amplitude of oscillation were investigated.

The added mass and damping of the ship motion were determined as well as the coupling moments (the pitching moments due to heaving velocity and acceleration). Comparisons are made between the various theoretical prediction procedures and these experimental results. The significance of the observed nonlinear damping forces is discussed.
\end{abstract}

\title{
INTRODUCTION
}

In the past five years the Transactions of the Society of Naval Architects and Marine Engineers has included a succession of papers dealing with the motion of ships in waves. These papers, by Weinblum, St. Denis, Pierson, and Korvin-Kroukovsky, present the tools necessary for the analytical determination of these motions.

Uniformly, the assumption is made that the forces on the body are of two types. The first is due to wave action and the remaining forces are proportional to the instantaneous position, velocity, and acceleration of the ship. This yields two linear, second order, differential equations with constant coefficients, which are simple to solve.

Unfortunately, these methods do not represent exact solutions to the boundary value problem. The analytical procedures require strong assumptions to be made in order to gain simplicity in the solution. Among these are the use of two-dimensional solutions, and the neglect of the free-surface influence, of nonlinear terms and of the speed dependence of the coefficients. Such an approximate procedure may be justified in practical ship problems, but the range of validity must be firmly established by adequate experimental investigations.

There have been few experimental studies wherein measured model motions have been compared with predicted motions using these analytical procedures. The little data available indicates that discrepancies do exist. No doubt, part of the problem involves the inherent experimental difficulties which make for poor accuracy in the measurements.

When discrepancies are revealed it is then difficult to decide whether to ascribe the fault to the form of the equations or to the particular coefficients used in the computation. This difficulty can be avoided by experimentally determining the coefficients. The body can be oscillated and either the forces or motions measured. The dependence of these coefficients on speed of advance, frequency, and amplitude of oscillation can be determined. This experimental procedure is quite analogous to that used in aerodynamic stability investigations wherein the wind tunnel balance, whirling arm and oscillator are used to measure the 
so-called static and rotary derivatives. These are nothing more than the coefficients in the equations of motion. Having this information, reliance can be placed on the coefficients, and the comparison between theoretical and experimental motions then becomes an adequate test of the linear equations. In the past, no thorough experimental study of this kind has been made on ship forms.

In this report only the first phase of a broader investigation will be described; namely, the forces and moments due to heaving motions. It is the purpose of the overall study to completely define the terms in the equations by experimental means, to evaluate the existing analytical procedures for determining these terms, and finally, to compare predicted and observed motions using these experimental results.

It has been most common in the past to use a free oscillation technique for such studies primarily due to its simplicity. However, there are difficulties in interpreting the data since the motion is not strictly periodic. Also, we are not assured of a given frequency of oscillation and, of course, the effect of amplitude cannot be determined.

The forced oscillation of the model overcomes these objections, with two alternative methods being possible. Either the model can be supported on a spring and oscillated, or it can be oscillated while supported on a stiff force balance. The latter method has been used because the amplitude of oscillation can be preset and the coupling forces and harmonic content can be directly measured.

At the outset, credit should be given Dr. Georg Weinblum for having led the way in investigations of this kind. It was through his efforts while associated with the David Taylor Model Basin several years ago that the Model Basin now possesses the mechanical oscillator, as well as the ship models used for these tests.

\section{THE MODEL}

The syrnmetrical model used in the present tests was one of a family constructed to mathematical lines defined by Weinblum. 1 The family has the general form

$$
\eta=\left[X(\xi)-v(\xi) v_{1}(\zeta)\right][Z(\zeta)]
$$

where the longitudinal, transverse, and vertical offsets of the hull, $x, y$, and $z$, are divided by the half-length, half-breadth, and draft to yield the nondimensional offsets, $\xi, \eta$, and $\zeta$. The equation of the waterplane is $\eta=X(\xi)$ and the equation of the midship section is $\eta=Z(\zeta)$. For the particular model used the form was described by

$$
\eta=\left[\left(1-\xi^{2}\right)-\left(\xi^{2}-\xi^{4}\right) \zeta^{10}\right]\left[1-0.3 \zeta^{8}-0.7 \zeta^{150}\right]
$$

The model constants are tabulated in Table 1 and the body plan is shown in Figure 1.

\footnotetext{
${ }^{1}$ References are liated on page 31.
} 
TABLE 1

Model Constants

\begin{tabular}{|l|cl|}
\hline Length & 136 & inches \\
Beam & 16 & inches \\
Draft & 6.4 & inches \\
Displacement & 316.6 & pounds \\
Midship Area Coefficient & 0.964 \\
Load Waterline Coefficient & 0.667 \\
Prismatic Coefficient & 0.655 \\
Block Coefficient & 0.632. \\
\hline
\end{tabular}

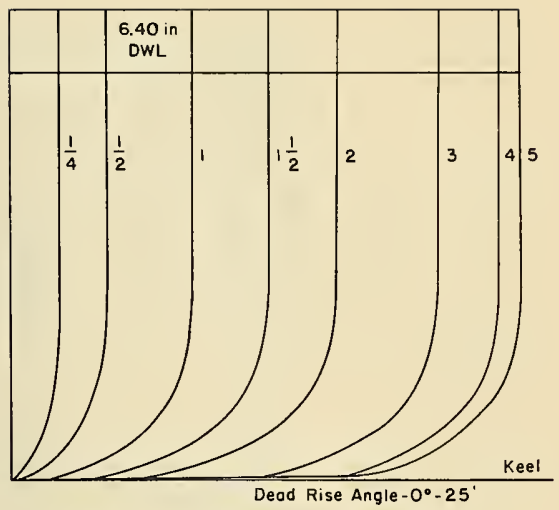

Figure 1 - Body Plan of the Model

The model was constructed as light as possible (about 80 pounds) and no attempt was made to ballast it to design displacement. This reduced to a minimum the inertia loading of the force balance during oscillation and allowed greater accuracy in determination of hydrodynamic force components.

\section{TEST EQUIPMENT}

The mechanical oscillator is mounted on the carriage over the deep-water basin. A d-c motor drives a Scotch yoke through an intermediate flywheel and 2-step gear reducer. The Scotch yoke converts rotation to linear motion and causes a vertical rigid strut to perform simple harmonic heaving oscillations. The oscillation frequency is varied by altering the motor speed and gear box setting, while oscillation amplitude can be altered by mechanical adjustment of the Scotch yoke mechanism.

A six-component strain-gage balance (Figure 2) is attached to the bottom of the oscillating strut and the model is attached to the balance. At midstroke of the oscillator the model is at the specified draft, keel parallel to the water surface, and the center of the symmetrical model is below the oscillating strut.

The six-component balance is positioned so that it directly measures lift and drag force and pitching moment about the center of the model. These three components are sensed by 4 active-arm bridge circuits and are recorded on a string oscillograph. In addition, a Schaevitz differential transformer provides a record of the heaving displacement. A sample record is shown in Figure 3. 


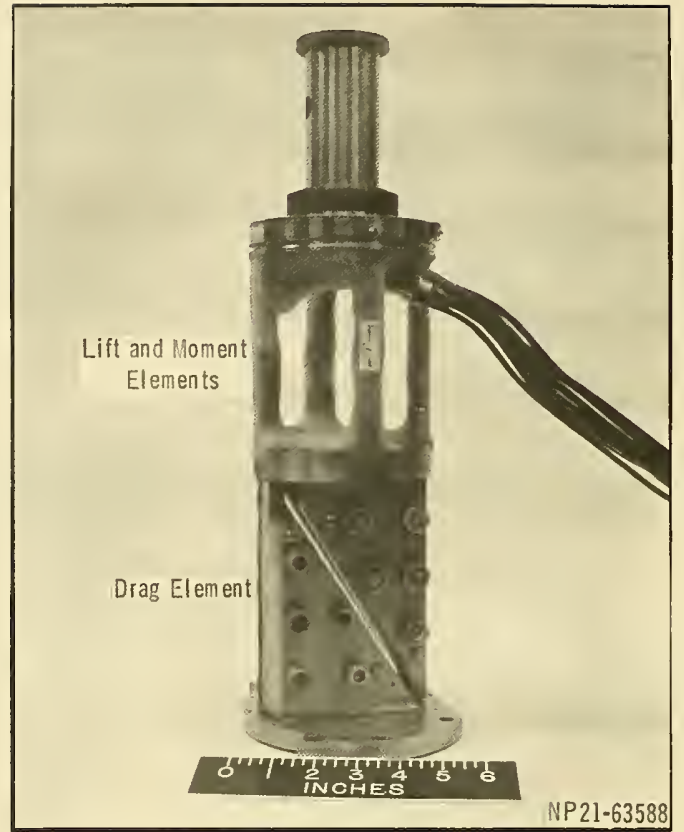

Figure 2 - The Six-Component Strain-Gage Balance

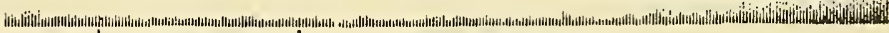
$\underset{4}{L}$ I second $\longrightarrow$ Timing Reference

Pitching Moment

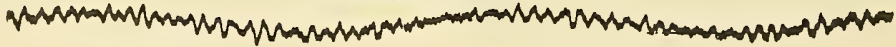
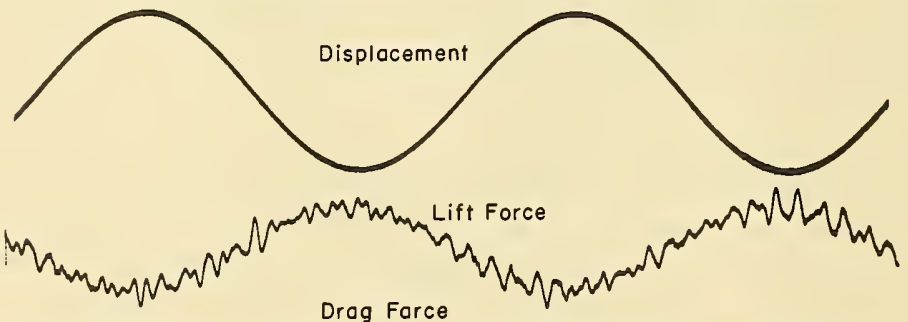

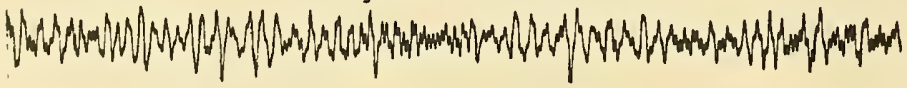

Figure 3 - A Sample Oscillograph Record 


\section{TEST PROGRAM}

It was necessary to determine the influence of forward speed and amplitude and frequency of oscillation on the hydrodynamic forces and moments.

Tests were run at $0,1,2,3$, and 4 knots, corresponding to Froude numbers of 0 to 0.35 . The amplitude of oscillation was $\pm 1 / 4, \pm 1 / 2$ or \pm 1 inch, and the frequency was varied from about $1 / 8$ to 3 cycles per second. It was hoped that the amplitude range was broad enough to establish the linear range of the equations of motion. The practical range of frequencies for ship motions at model scale would not exceed 2 cycles per second but the higher frequencies are of interest in establishing the validity of several theoretical methods of determining ship damping.

\section{METHOD OF ANALYSIS}

A surface ship model is constrained to oscillate sinusoidally, $z=z_{0} e^{i \omega t}$, at frequency $\omega$ and with amplitude $z_{0}$. It will be assumed that the ship experiences forces and moments proportional to the instantaneous displacement and its first and second derivatives. A balance, located between the ship and the oscillating support senses the lift force and pitching moment on the ship, $F=F_{0} e^{i(\omega t-\delta)}$ and $M=M_{0} e^{i(\omega t-\alpha)}$. The force and moment equations are

$$
\begin{gathered}
A \ddot{z}+B \dot{z}+C z=F_{0} e^{i(\omega t-\delta)} \\
d \ddot{z}+e \dot{z}+f z=M_{0} e^{i(\omega t-\alpha)}
\end{gathered}
$$

Substituting $z=z_{0} e^{i \omega t}$ into these equations yields

$$
\begin{aligned}
& \left(-A \omega^{2}+C\right)+i \omega B=\frac{F_{0}}{z_{0}} e^{-i \delta} \\
& \left(-d \omega^{2}+f\right)+i \omega e=\frac{M_{0}}{z_{0}} e^{-i \alpha}
\end{aligned}
$$

Equating real and imaginary parts of [2], we obtain

$$
\begin{gathered}
-A \omega^{2}+C=\frac{F_{0}}{z_{0}} \cos \delta \\
\omega B=\frac{-F_{0}}{z_{0}} \sin \delta
\end{gathered}
$$

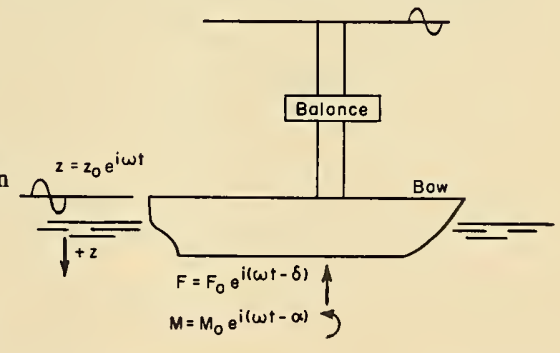

Figure 4 - Schematic Diagram of the Model and Balance 


$$
\begin{gathered}
-d \omega^{2}+f=\frac{M_{0}}{z_{0}} \cos \alpha \\
\omega e=\frac{-M_{0}}{z_{0}} \sin \alpha
\end{gathered}
$$

In [1a] the coefficient $A$ is recognizable as the virtual mass, the sum of the mass suspended beneath the balance $m$ and the hydrodynamic added mass $m_{a}$. The damping force $B \dot{z}$ represents, primarily, the energy dissipated by the system in progressive gravity waves. The coefficient $C$ is the effective "spring" constant or buoyant force coefficient. For the range of amplitudes used in these tests, the ship is essentially wall-sided so that $C$ can be assumed constant.

Solving for the unknown hydrodynamic coefficients in terms of known or measurable quantities, we obtain,

$$
\begin{gathered}
m_{a}=\frac{C-\frac{F_{0}}{z_{0}} \cos \delta}{\omega^{2}}-m \\
B=\frac{-\frac{F_{0}}{z_{0}} \sin \delta}{\omega}
\end{gathered}
$$

The hydrodynamic moments imposed on the ship by the heaving motion are given in Equation [1b] and results in the coupling of the heaving and pitching motions. Since the model in these tests has fore-and-aft symmetry and nearly wall sides, it can be assumed that there is negligible buoyancy moment due to heave so that $f=0$.

Solving for the cross-coupling coefficients in Equation [3b]

$$
\begin{aligned}
& d=-\frac{\frac{M_{0}}{z_{0}} \cos \alpha}{\omega^{2}} \\
& e=-\frac{\frac{M_{0}}{z_{0}} \sin \alpha}{\omega}
\end{aligned}
$$


In the actual experiment, the motion $z$, of the model cannot be measured directly. In. stead the motion of the oscillating strut above the balance is recorded. Since the balance cannot be infinitely stiff, these two motions may differ in amplitude and phase. This is particularly true at high frequencies; that is, high relative to the natural frequency of the system. In Appendix A the dynamic system is analyzed, considering the balance to be a spring, and correction factors are obtained which allow the above analysis to be used.

\section{TEST RESULTS}

\section{FORCE COEFFICIENTS}

In order to determine the added mass and damping coefficients it has been shown that the complex ratio, $F / z$ or $F_{0} / z_{0} e^{-i \delta}$, must be determined.

The variation of $F_{0} / z_{0}$ with frequency for constant speeds of $0,1,2,3$, and 4 knots and displacement apmlitudes of $1 / 4,1 / 2$, and 1 inch are shown in Figure 5.

Figure 6 shows the complementary plots of $\delta$, the phase angle by which the force lags the displacement. At zero speed, for low frequencies, these data scattered excessively and so are not shown in Figure 6a. It is believed that this is due to wave reflections from the basin walls. At low frequencies, the high wave celerity makes it difficult to conclude a test before significant reflections are felt at the body. The phase angle at 3 and 4 knots at high frequencies is not shown due to excessive noise in the trace caused by carriage vibration.

The dynamic system represented by the model supported on the balance has a natural frequency of about $70 \mathrm{rad} / \mathrm{sec}$. Random disturbances cause the model to oscillate at this frequency, the amplitude increasing as the carriage speed increases. In addition, the oscillator is mounted on a carriage which has its own vibratory modes and which is excited by the oscillating model and the motion of the carriage. Thus, the model vibrates at the oscillator frequency and in accordance with these extraneous influences. These additional high frequencies appear in the force and moment records and necessitate hand fairing of the traces in order to extract the true fundamental component.

The damping coefficient $B$ is proportional to the sine of the phase angle $\delta$. At high frequencies, $\delta$ approaches $180 \mathrm{deg}$ and great accuracy is required in the phase angle determination in order to obtain a reasonably accurate coefficient. It was found necessary, for frequencies above $7 \mathrm{rad} / \mathrm{sec}$, to determine all phase angles shown in Figure 6 by harmonic analysis using a "Runge Schedule.", 2

The dynamic correction described in Appendix A has already been applied to the data shown in Figures 5 and 6.

The physcial explanation of the shape of the curves in Figures 5 and 6 can be made as follows: the total force on the model is the sum of three forces; buoyancy, damping, and inertia. The buoyancy force is independent of frequency. The damping force is proportional to frequency, and leads the buoyancy force by $90 \mathrm{deg}$, while the inertia force is proportional 
Figure 5 - Plots of $F_{0} / z_{0}$ versus Frequency at Various Speeds

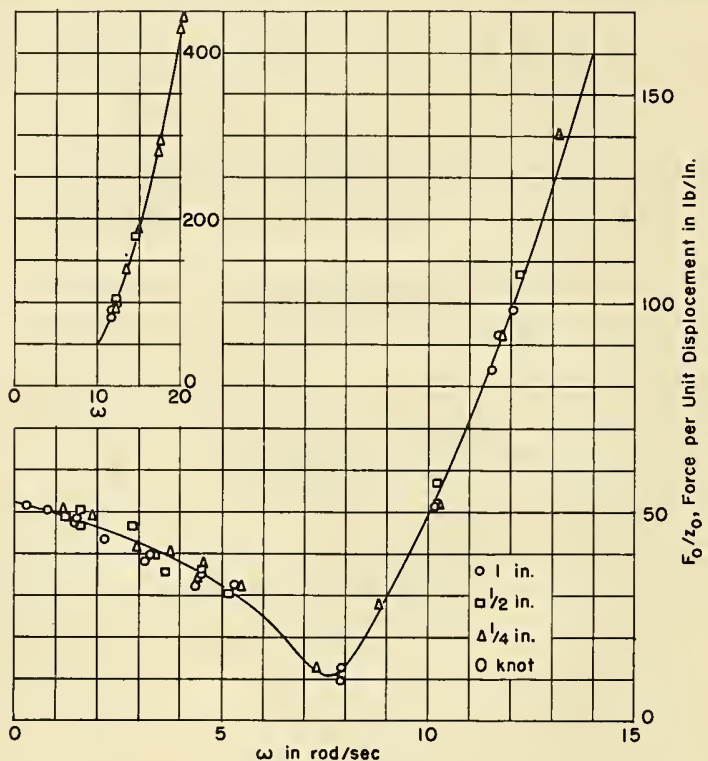

Figure 5a-0 Knot

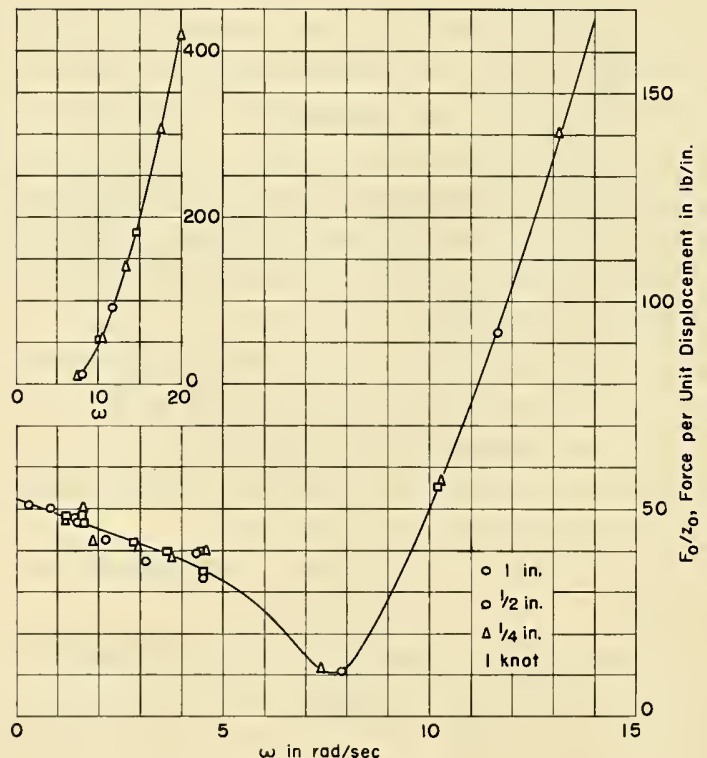

Figure 5b - 1 Knot 


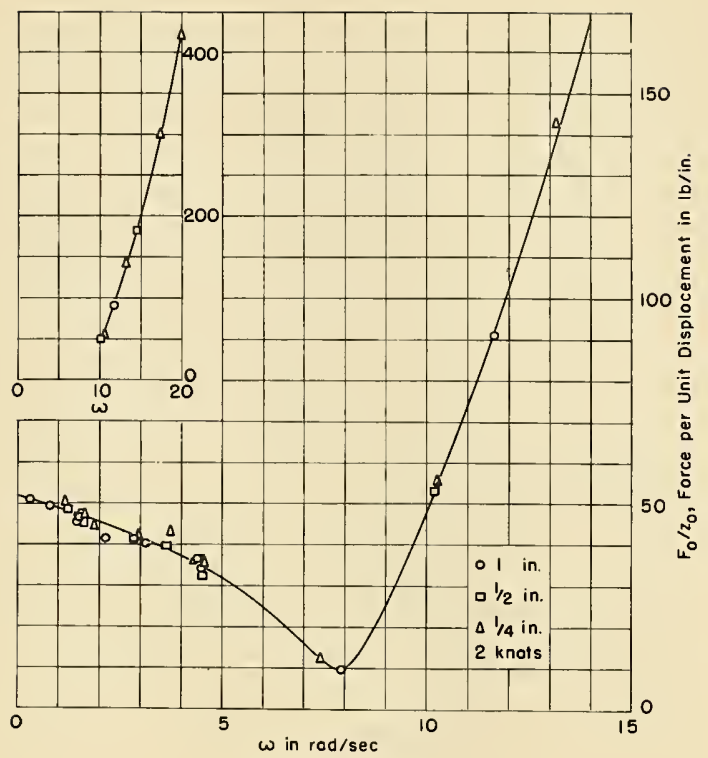

Figure 5c- 2 Knots

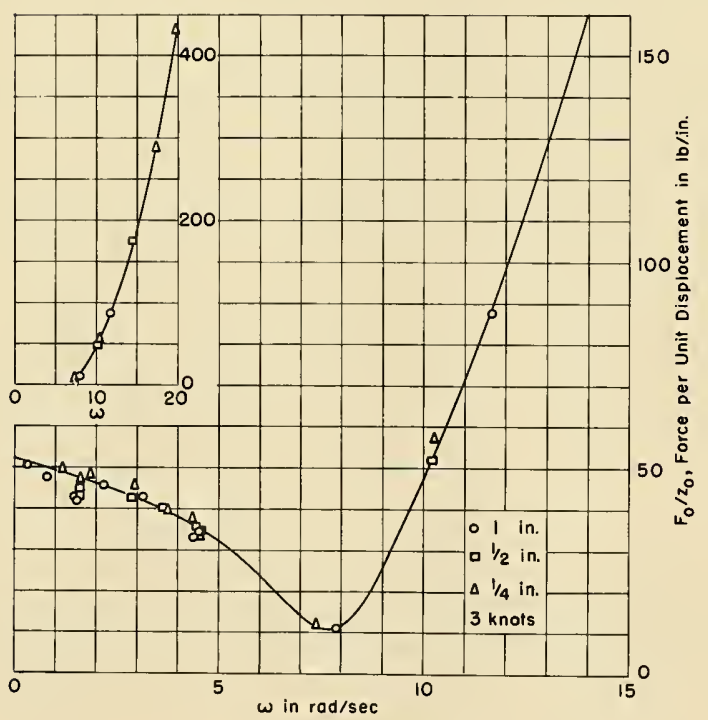

Figure 5d - 3 Knots 


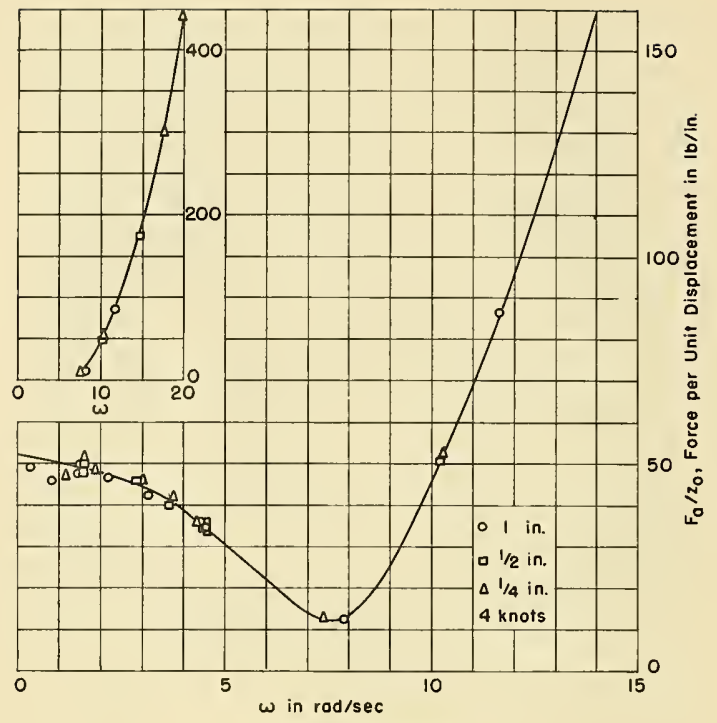

Figure 5e -4 Knots

to $\omega^{2}$ and leads the buoyancy force by $180 \mathrm{deg}$ (Figure 7). Assuming the coefficients $A, B$, and $C$ to be approximately constant and the damping to be small, it can be seen that at very low frequencies the total force is essentially all buoyancy force and the phase angle is very small. As the frequency increases the inertia force increases and cancels part of the buoyancy force so that the total force drops and the phase angle increases toward $90 \mathrm{deg}$. When the two are equal, the total force curve is at its minimum, the phase angle is $90 \mathrm{deg}$. and we see the pure damping force in the record. As frequency increases, the inertia force increases as $\omega^{2}$ so that the total force at high frequencies is essentially the inertia force. The total force rises sharply and the phase approaches $180 \mathrm{deg}$.

The data shown in Figures 5 and 6 are substituted in Equation [4a] to obtain the added mass $m_{a}$ and damping coefficient $B$. Below $\omega=7$, values obtained from the faired curves of $F_{0} / z_{0}$ and $\delta$ were used. Above $\omega=7$, however, due to the sensitivity to small errors in $\delta$, the measured values for each run were combined to yield $m_{a}$ and $B$ and then these results were faired.

Figure 8 is a plot of an added mass coefficient $k_{z}=\frac{m_{a}}{\Delta / g}$ where $\Delta$ is the displacement of the model and $g$ is the acceleration of gravity. In Figures 5 and 6 no systematic amplitude effects were observed so that the added mass in Figure 8 represents all amplitudes used in the tests. At low frequencies, the added mass appears to decrease with increased forward speeds. However, at higher frequencies for Froude numbers $0,0.09$, and 0.18 , there does not appear to be any variation of $m_{a}$ with speed. 
Figure 6 - Plots of Phase Angle between Force and Displacement at Various Speeds
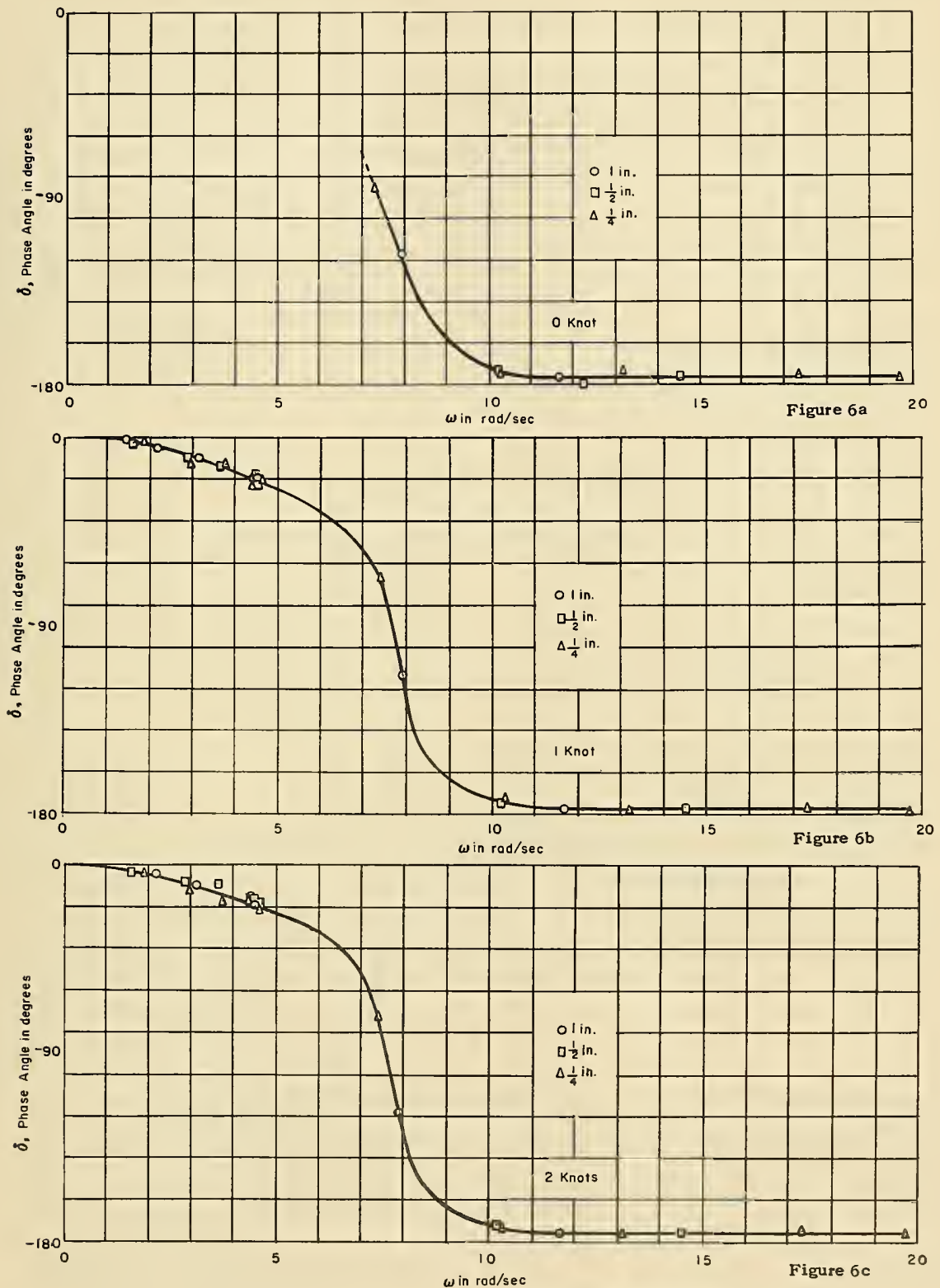


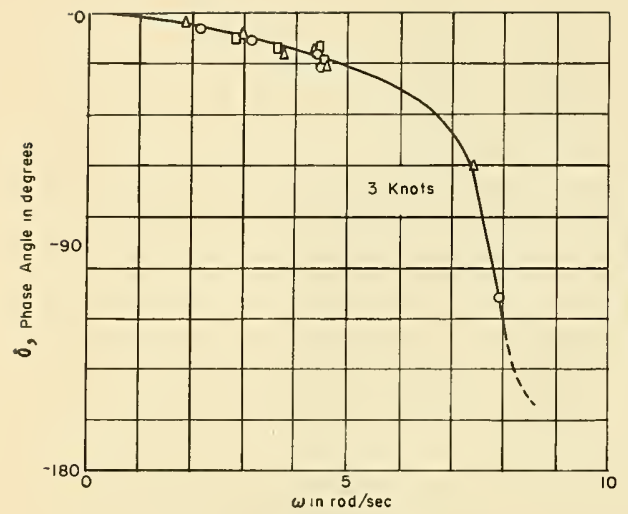

Figure 6d

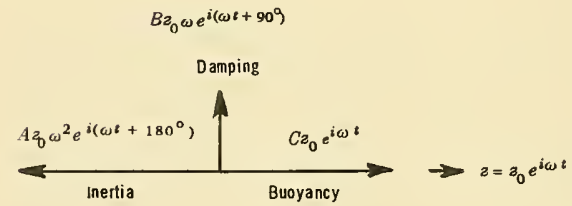

Figure 7 - Vector Presentation of Buoyancy, Damping, and Inertia Forces

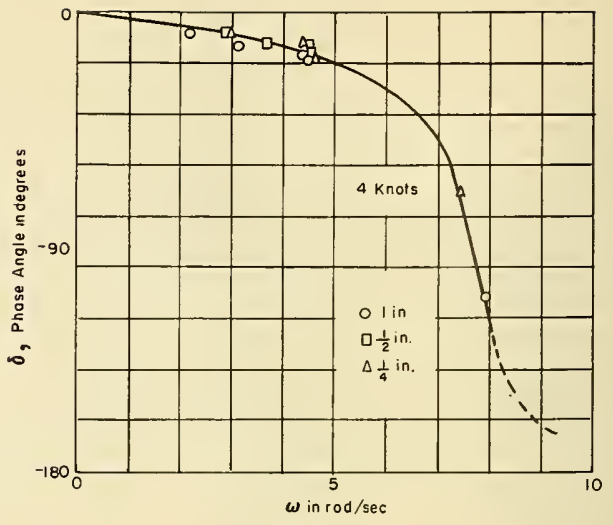

Figure $6 \mathrm{e}$

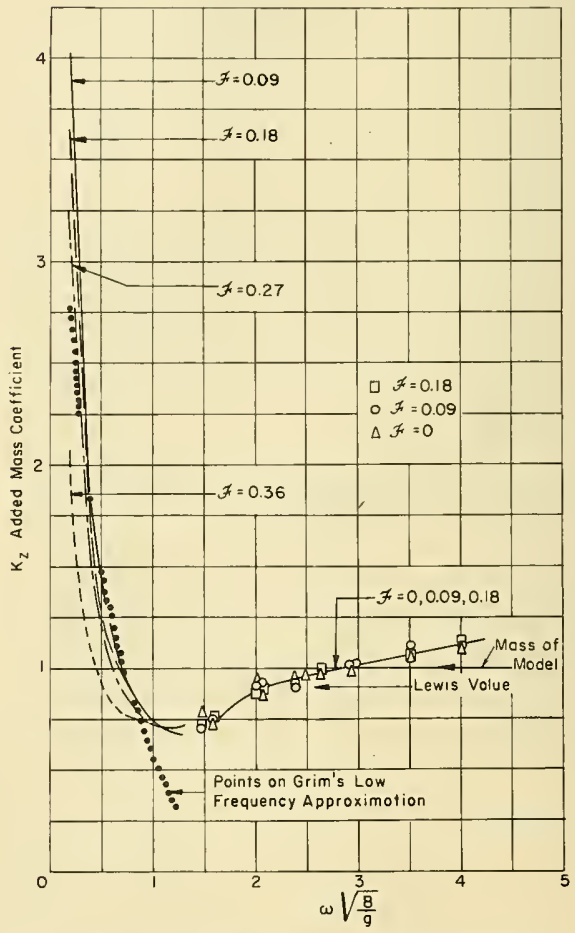

Figure 8 - The Variation of Added Mass Coefficient with Frequency 


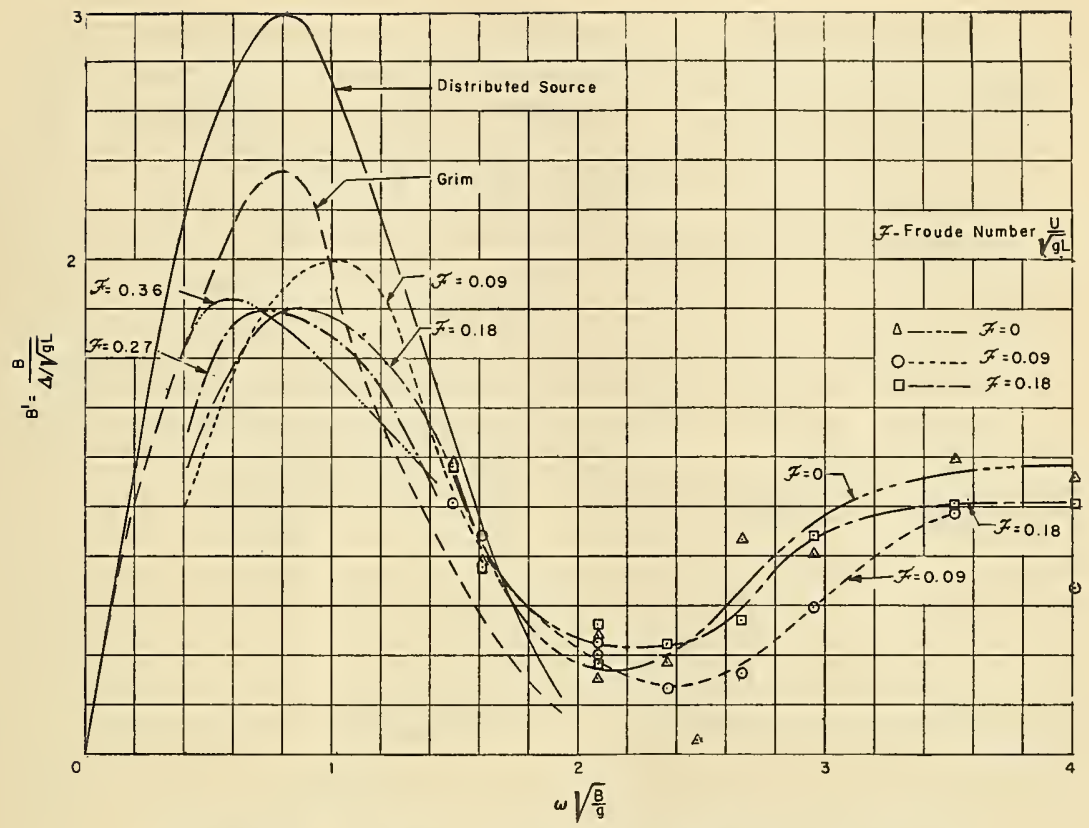

Figure 9 - The Variation of Damping Coefficient with Frequency

A damping coefficient defined by $B^{\circ}=\frac{B}{\Delta / \sqrt{g L}}$, where $L$ is the length of the model, is given in Figure 9 for nondimensional frequencies between 0.4 and 4 . Below $\omega \sqrt{B / g}=0.4$, the accuracy of $B$ is very poor due to the smallness of the phase angle, $\delta$.

The damping did not show any dependence on the amplitude of motion as was true for the added mass. A decrease in forward speed causes the peak in the damping curve to move to higher frequencies although it has little effect on the maximum value.

\section{MOMENT COEFFICIENTS}

Equation [4b] indicates that the moment coefficients $d$ and $e$ are determined from the amplitude ratio of pitching moment to displacement, $M_{0} / z_{0}$, and the phase angle $\alpha$ by which the moment lags the displacement. The amplitude ratio $M_{0} / z_{0}$ is presented in Figure 10, the dynamic correction described in Appendix A having already been applied. The phase angle is shown in Figure 11.

Since the model had fore-and-aft symmetry, tests were run both east and west. Although the hydrodynamic moments must be the same in both directions the phase angle clearly is 


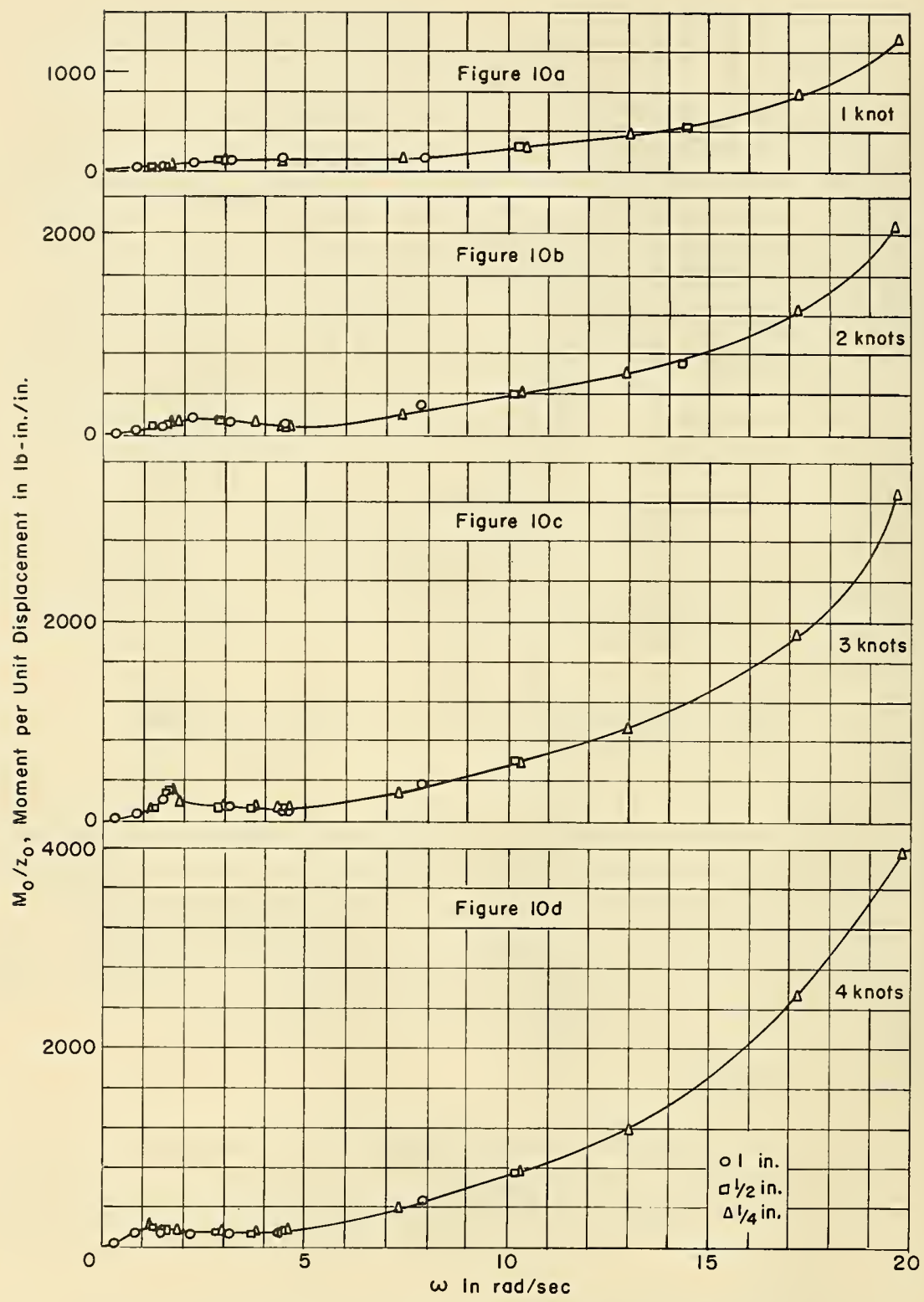

Figure 10 - Plots of $M_{0} / z_{0}$ versus Frequency at Various Speeds 


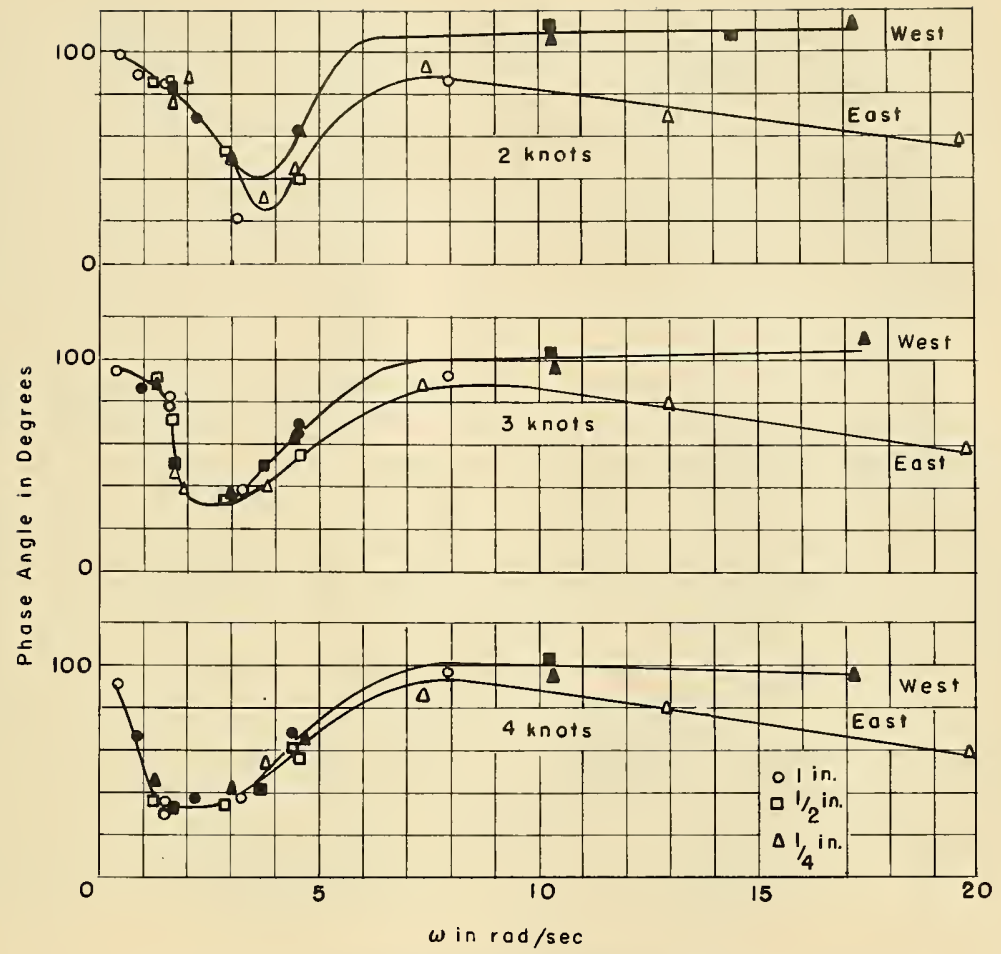

Figure 11 - $\alpha$, Phase Angle Between Moment and Displacement at Various Speeds

different at higher frequencies. This is due to a mass unbalance in the model which yields a moment signal proportional to the square of the frequency. This moment must change sign relative to the apparent bow as the direction of travel reverses. Thus it either adds or subtracts from the hydrodynamic moment so that the phase angle for travel east and west is different. The amplitude ratio is essentially unaltered.

The magnitude of this extraneous inertia loading can be obtained from the moment versus frequency curve at zero speed since at this speed no hydrodynamic moment can exist. It was found that the unbalance was equivalent to a weight of 32 pounds at an arm of 1 foot.

In Figure 11 it was necessary to draw the two curves ("east" and "west") so that when the inertia moment correction is made to the data in applying Equation [4b], the east and west tests will yield the same coefficients $d$ and $e$. That is, the two curves are a best fit to the data spots while being consistent with each other. At low forward speeds, the accuracy of the hydrodynamic moment is low since its magnitude is small relative to the 


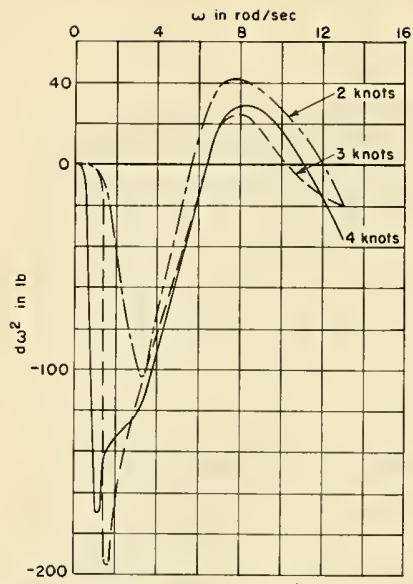

Figure 12a - The Variation of $d \omega^{2}$ with Frequency

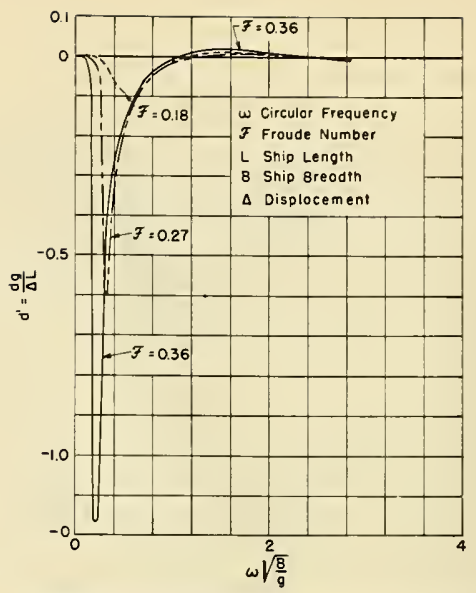

Figure 12b - The Variation of $d$ with Frequency

Figure 12 - The Variation of the Coefficient $d$ with Frequency

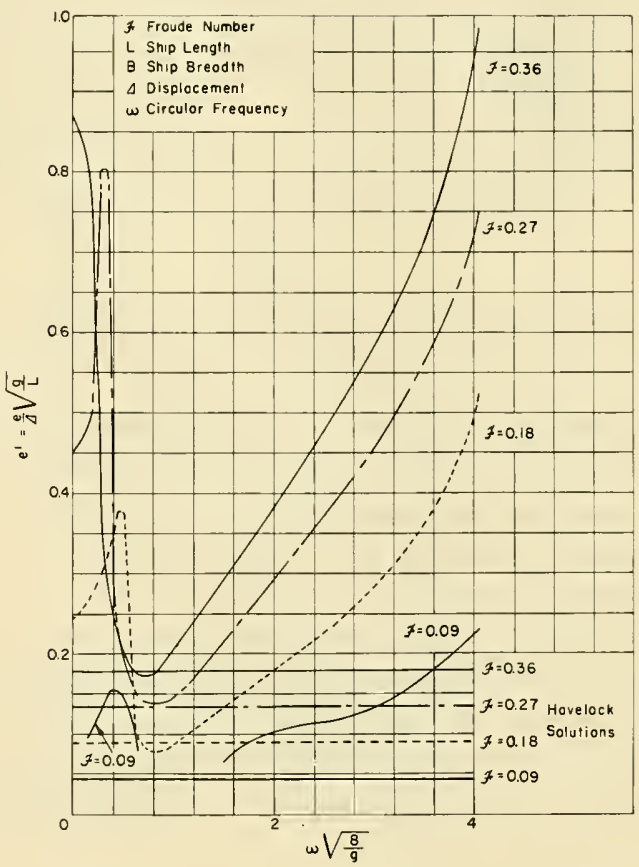

Figure 13 - The Variation of the Coefficient $e$ with Frequency 
inertial correction. Phase angles at 1 knot are not presented for this reason and also because the moment signal was unsteady in the neighborhood of $\omega=4 \mathrm{rad} / \mathrm{sec}$ at $1 \mathrm{knot}$.

Applying Equation [4b] to these data yields the coefficients $d$ and $e$. In Figure 12a, the product $d \omega^{2}$ (which is proportional to the moinent on the model) has been plotted in order to better present the data at high frequencies. In Figure $12 \mathrm{~b}$ a nondimensional moinent coefficient $d^{\prime}=\frac{d g}{\Delta L}$ has been used. The nondimensional coefficient $e^{\prime}=\frac{e}{\Delta} \quad \sqrt{g / L}$ is given in Figure 13. Unlike the coefficient $d^{\prime}$, the value of $e^{\prime}$ at 1 knot is given because it is insensitive to errors in phase angle.

\section{SECOND HARMONICS IN THE LIFT FORCE}

It is commonly assumed in ship-motion problems that the hydrodynamic damping varies as the first power of the velocity. This is true of bodies moving in a fluid at relatively low velocities but at larger velocities a dependence on the second power of velocity more nearly describes the physical phenomenon. Such an assumption destroys the linearity of the system and necessarily complicates the solution of the problem. Therefore, it would be of interest to examine the lift data in these experiments in order to determine the validity of the usual linearizing assumptions.

The existence of square law damping would be indicated by significant second harmonic content in the lift force trace. This can be shown as follows: assume a damping force of the form $B_{2}(d z / d t)^{2}$. Then, if the ship is constrained to perform heaving oscillations $z=z_{0} \sin$ $\omega t$, the measured damping force will be

$$
B_{2} \dot{z}^{2}=B_{2} z_{0}^{2} \omega^{2} \frac{1+\cos 2 \omega t}{2}
$$

Thus the observed amplitude of second harmonic in the lift force will be

$$
\frac{B_{2} z_{0}^{2} \omega^{2}}{2}
$$

Unfortunately, the present experiments do not lend themselves readily to such meas. urements. The second harmonie is largely masked by the inertia, buoyancy, and fundamental of the damping force. It only becomes visually apparent on the records at the frequency where the inertia and buoyancy forces cancel one another. If one were to redesign the experiment to accurately determine this component, the fundamental should be filtered-out and the second harmonic amplified greatly.

All lift curves at frequencies above $7 \mathrm{rad} / \mathrm{sec}$ and at speeds of 0,1 , and 2 knots were harmonically analyzed in order to obtain accurate phase lags at the fundamental frequency. This analysis also yielded the amplitude of the second harmonic. In addition a few tests at 
lower frequencies were analyzed similarly. The second harmonic forces resulting from the 1/4-inch oscillations were to small to be analyzed. As a result only 19 tests spots were obtained.

It was found when the widely scattered force data was divided by $z_{0}^{2} \omega^{2} / 2$ that the resulting damping coefficient $B_{2}$ was scattered but remained within an interval of 0.06 to 0.16 lb-sec ${ }^{2} /$ in $^{2}$, as shown in Figure 14. The accuracy of the data was too poor to define a frequency, amplitude, or speed dependence. In Figure 15 the number of test points in an interval is shown in a bar graph. We are compelled at this stage, to assume a single "best" value of $0.11 \pm 0.05$ for all speeds, frequencies, and amplitudes.

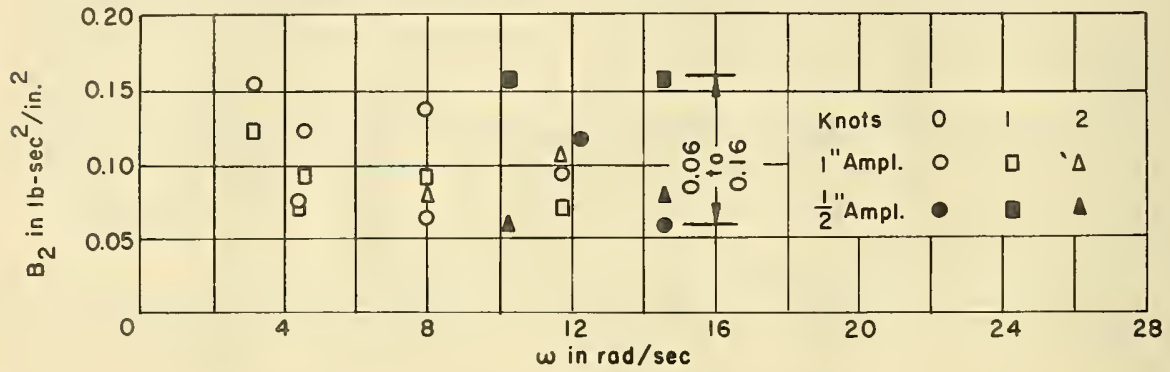

Figure 14 - The Nonlinear Damping Coefficient $B_{2}$

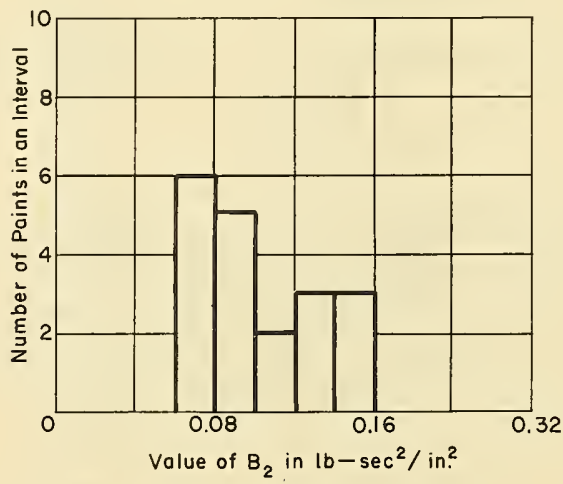

Figure $15-B_{2}$, The Number of Points in an Interval 


\section{DISCUSSION}

\section{PREDICTION OF DAMPING-IN-HEAVE}

Recent papers on ship motions have utilized the principle that the damping of the vertical motion of a ship's section can be related to the waves generated by a suitable distribution of pulsating sources along its surface. The attractive feature of this method is its essential simplicity.

One of the characteristics of this solution is the existence of an infinite succession of the values of the frequency at which the damping is zero. This phenomenon is due to interference effects between the wave systems generated on both sides of the body.

Ursell $^{3}$ has pointed out that at higher frequencies, when the draft is several times the wavelength, there is a shielding effect and there cannot be any interference. In fact, he has demonstrated that the exact solution does not reveal any such zeros and showed that the pulsating source technique is valid only at low frequencies. Ursell was able to find the exact solution for several oscillating cylindrical forms.

Grim $^{4}$ has found a nearly exact solution for a wider class of two-dimensional forms. The section is reflected about the waterplane and the potential for this double body in an infinite medium is found. To this is added a potential such that the sum of the two satisfies the free-surface condition. To these are then added a sum of other potentials so that the total still satisfies the free-surface condition and satisfies the boundary conditions at certain points on the body. Only a few such terms are found necessary in the calculation. From these results the added mass and the damping can be calculated. Grim's results for the added mass compare very closely with the exact solutions of Ursell. No multiple zeros are found in the damping.

Grim has computed the wave amplitude (and consequently the damping) of a class of ship-like forms of beam-draft ratios of $3,2,4 / 3$, and 0.4 with varying fullness. An effort will be made to compare these results with the experimental values. A procedure is used which is analogous to that used by Prohaska ${ }^{5}$ for finding the added mass.

It is assumed that ship-like forms having the same beam-draft ratio and fullness would have the same damping characteristics. Thus for any section, Figures 9, 10, 11, and 13 of Reference 4 can be used, with suitable interpolation, to find the damping of each section of the model. Integrating these results along the length of the body gives the total damping. No correction was made for the three-dimensional nature of the flow. Grin suggests that such a correction is not necessary for ship forms when the frequency is large enough, say $\omega^{2} L / g>6$. For the model being tested, this corresponds to $\omega>4 \mathrm{rad} / \mathrm{sec}$.

In Figure 9 the experimental damping coefficient $B$ for 0,1 , and 2 knots is given. Also shown is the damping as computed by two methods; using the Grim curves and the distributed source technique. Both the Grim method and source method over estimate the damping but the 
Grim method yields a better approximation. It should be noted that a three-dimensional correction, especially at the lower frequencies, would tend to reduce the damping and so improve the prediction.

Both theoretical procedures, although differing on the existence of zeros, predict very small damping at higher frequencies. As mentioned in an earlier section the accuracy of the measured damping coefficient at higher frequencies is severely limited by inaccuracies in the phase-angle determination. Therefore, it is not possible for the experiment to resolve the question concerning multiple zeroes.

At the higher frequencies the experiments show the damping falling toward zero and then increasing with the increasing frequency. In spite of the experimental error, there is clear evidence that at higher frequencies this increase with increasing frequency does in fact exist. This rise in damping cannot be explained by means of the suggested theories, for at frequencies above $\omega \sqrt{B / g}=2.5$ they would predict essentially zero damping. One might speculate that another damping law is indicated at high frequencies.

\section{PREDICTION OF ADDED MASS}

The common method used for prediction of the added mass of surface ships for vertical motion is based on the familiar work of Lewis ${ }^{6}$. By suitable conformal mapping, the circle is transformed into a ship-like section which has been reflected about the waterplane. The added mass of the ship section is assumed to be one-half that of the ship and image together in an infinite medium. This procedure is theoretically correct only at high frequencies since the influence of gravity on the free surface is neglected. The section characteristics are integrated over the length of the body and are then corrected by a factor to account for the threedimensional effects. This factor is chosen to be the ratio of the exact solution and the integrated two-dimensional results for an ellipsoid whose three axes are equal to the principal dimensions of the ship plus image. Thus the procedure yields an added mass which is independent of frequency, amplitude of oscillation, and speed of advance.

Prohaska ${ }^{5}$ extended this procedure by assuming that the added mass of a ship section was dependent on the fullness and beam-to-draft ratio of the section alone. The Lewis transformation was used to set up this dependency and all other forms were assumed to follow the same law. Some experimental evidence was used to substantiate this assumption.

The Lewis-Prohaska procedure was used for the model being tested and the results compared with the experiments. Since the calculations involved two distinct processes (that is, the integration of section characteristics and the correction for three-dimensional effects), it is not possible to ascribe differences between calculation and theory to a shortcoming in either step. In Figure 8, it is clear that the constant value obtained in the calculation cannot represent the strong frequency dependence of the added mass. In fact the added mass 
does not asymptotically approach the Lewis value at high frequencies as might be expected. It is seen to be 20 percent higher than the Lewis value at the highest frequency shown and is still rising.

The two-dimensional solutions obtained by $\mathrm{Grim}^{4}$ can be used to obtain the frequency dependence of the added mass. Grim's results show the added-mass coefficient dropping sharply from infinity to a minimum value as the frequency is increased and then rising asymptotically toward the high frequency solution for the section reflected in the waterplane. This general form of the curve was also obtained in Ursell's exact solution for the circular cylinder. Observing the test results in Figure 8, we see that the experimental curve for a threedimensional form has this same general shape. Similarly, the experimental results of Haskind and Riman ${ }^{7}$ show this sane trend.

Grim has indicated that at low frequencies the added mass of any section can be approxi-

mated by $-\frac{4}{\pi} \rho b^{2} \ln \xi$ where the frequency parameter $\xi$ is $\frac{b \omega^{2}}{g}, b$ is the half-breadth of the section, $\rho$ is the mass density, and $\omega$ is the circular frequency. Comparison of this approximate formula with Grim's exact solution (Figures 1 and 2 of Reference 3 ) would indicate that the approximation is good up to values of about $\xi=0.4$.

The Grim approximation has been applied to the model under test and the two-dimensional results integrated over the length of the body. The resulting added-mass values are shown in Figure 8. The small three-dimensional end correction has not been applied to the curve.

It can be seen that the sharp drop of added mass with increasing frequency is adequately represented by this approximation up to a frequency $\omega \sqrt{B / g}=0.8$ or $\xi=1 / 3$. This confirms the utility of the Grim method.

The two-dimensional theoretical solutions do not reveal any dependence of added mass on forward speed. The experimental results indicate the speed effects are negligible except at very low frequencies where the added mass at 4 knots is lower than that at slower speeds. However, the data at these low frequencies is not considered accurate enough to warrant any definitive statements on speed dependence.

\section{COUPLING OF HEAVE AND PITCH}

Havelock ${ }^{8}$ has recently investigated the coupling of the heaving and pitching motions for a body with forward velocity. A long spheroid half immersed in a uniform stream executes small heaving and pitching oscillations. A rigid wall surface condition is satisfied and damping is neglected. He finds a heaving force equal to - $p M U \dot{\theta}$ and a pitching moment equal to $q M U \dot{z}$ where $M$ is the displaced mass, $U$ is the forward velocity, $\dot{\theta}$ is the pitching velocity, $\dot{z}$ is the heaving velocity, and $p, q$ are positive coefficients. Calculations show that $p$ and $q$ are approximately 1 and $1 / 2$ respectively. 
Haskind ${ }^{9}$ has found similar terms in the equations of motion although the constants $p$ and $q$ are different. This results from the imposition of another surface condition, namely that $\phi=0$ on the surface. For the elongated spheroid $p$ and $q$ equal $1 / 2$.

A recent paper by Korvin-Kroukovsky, ${ }^{10}$ using the "source" wethod for computing ship damping, indicated that a ship with fore-and-aft symmetry and a forward velocity can experience no pitching moment due to heaving velocity. As Havelock suggests, the asymmetry in the flow should result in such a coupling moment. The data appearing in Figures 12 and 13 confirm this thesis.

The present experiments offer an opportunity to check the values of the theoretical coupling terms given by Havelock and Haskind. Havelock calculated the coefficient desig. nated as $q$ to be about $1 / 2$. Using the mass of a floating spheroid of length and displacement equal to that of our model, Havelock's expression, $e=q M U$, has been nondimensionalized and plotted in Figure 13. The magnitude of these frequency-independent curves proportional to the forward speed correspond to the minimum point of the experimental curves. However, the experiments indicate a very strong frequency dependence not indicated by the theory. The steep rise at low frequencies appears to coincide with a velocity-wave celerity ratio of $1 / 4$ where the character of the waves generated by the oscillating body is known to change markedly. 11

Havelock neglects any pitching moment due to heaving acceleration whereas Haskind finds this term to be zero for a symmetrical model under way. However, our experiments indicate that such a moment, represented by the term $d \ddot{z}$ in the equations of motion, Equation [1b], does exist. In Figure 12 the coefficient $d$ is shown to be sharply peaked at low frequencies, the amplitude of the peak increasing with increasing speed as would be expected. However, at higher frequencies, the coefficient is very small and may be unimportant when compared to inertia moments.

It is not possible to immediately assess the importance of these coupling terms on the motion of a ship in a seaway. Havelock has computed the alteration in the frequencies of free oscillation due to these terms and found them to be only slightly altered even at high speeds of advance. However, this may not be a sufficient criterion to judge the effect on the motions. The answer awaits a detailed study of the solutions of the coupled equations of motion. Such a study could ideally be performed by analogue computer techniques.

\section{HASKIND-RIMAN EXPERIMENTS}

Haskind and Riman ${ }^{7}$ have performed experiments which are similar to those described in this report. A symmetrical model with V-sections was supported on a spring whose upper end was constrained to oscillate harmonically in heave. The damping and added mass of the heaving model at zero speed of advance was computed from the relative motions of the top and bottom of the spring. The damping coefficient was found to be independent of oscillation amplitude and its frequency dependence is shown in Figure 16. 


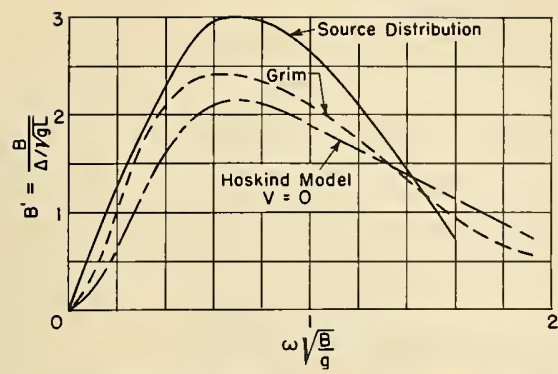

Figure 16 - The Variation of Damping Coefficient with Frequency for the Haskind Model
These data afford another opportunity to compare the Grim method and source method for computing damping. Again, the sections of the Haskind-Riman model were compared with the forms derived by Grim. Sections of equal fullness and beam-draft ratio were assumed to have equal damping. Suitable interpolation and integration over the body length gave the curve shown in Figure 16. Distributing pulsating sources on the surface of the sections, computing the waves generated and therefore the damping, and then integrating over the length gave the curve marked "Source Distribution." Again, as in the case of the present experiments, the Grim method is seen to yield a closer prediction of the damping although both methods predict excessive damping. Application of a threo-dinensional correction would improve the correlation.

\section{QUADRATIC DAMPING OF THE HEAVING OSCILLATION}

It has been shown earlier that a measurable amount of second harmonic content is present in the lift-force traces and that this could be attributed to the existence of quadratic damping of the heaving oscillation. It is intended at this point to evaluate its effect on the motions of a surface ship.

The method of Kryloff and Bogoliuboff ${ }^{13}$ allows one to find an approximate solution to nonlinear equations of the form

$$
\ddot{z}+\nu^{2} z+\epsilon f(z, \dot{z})=0
$$

where the motion is nearly sinusoidal.

The free oscillations of a heaving ship, assuming both linear and quadratic damping, can be written

$$
\ddot{z}+\nu^{2} z+\frac{B_{1}}{m} \dot{z}+\frac{B_{2}}{m}(\operatorname{sgn} \dot{z}) \dot{z}^{2}=0
$$

If the damping forces are small compared to the restoring forces, the approximate solution has been given ${ }^{12}$ as

$$
z=a \sin (\nu t+\phi)
$$


where

$$
a=\frac{\frac{B_{1}}{2} \frac{a_{0}}{\frac{4 \nu B_{2} a_{0}}{3 \pi}+\frac{B_{1}}{2}} e^{-\frac{B_{1}}{2 m} t}}{1-\frac{4 \nu B_{2}}{3 \pi} \frac{a_{0}}{\frac{4 \nu B_{2} a_{0}}{3 \pi}+\frac{B_{1}}{2}} e^{-\frac{B_{1}}{2 m} t}}
$$

and $a=a_{0}$ when $t=0$.

Setting $B_{2}=0$ we obtain the usual solution to the linear damped equation

$$
a=a_{0} e^{-\frac{B_{1}}{2 m} t}
$$

The decay curves for free heaving oscillations of the model under test have been calcuIated for several initial displacements, with and without quadratic damping. The curves were computed at $\nu=6.1$, the natural frequency in heave, using the measured damping coefficients.

In Figure 17 the amplitudes of successive peaks have been plotted for 1-, 2•, and 3-inch initial displacements. The dashed curves include the quadratic damping while the solid curves do not. It can be seen that the neglect of nonlinear damping becomes more serious as the initial amplitude increases. After the fourth peak, when all the amplitudes are small, all curves are essentially parallel. This indicates that when the motion is small enough they have the same logarithmic decrement or ratio of the amplitude of successive peaks. The rate of decay is then governed by the linear damping coefficient and the quadratic damping influence is negligible.

For this particular model, the peak of the linear damping curve occurs at about the natural frequency in heave. This would tend to minimize the influence of nonlinear damping. No general conclusions should be drawn since this may be coincidental and other forms may show greater nonlinear effects at the frequency of free oscillation.

For forced oscillations, the method of "equivalent linearization" can be used. The nonlinear damping term $B_{2}$ (sgn $\dot{z}$ ) $\dot{z}^{2}$ is replaced by a linear term such that the work dissipated per cycle by each term is the same; that is,

$$
\int_{0}^{T}\left(B_{e} \dot{z}\right) \dot{z} d t=\int_{0}^{T}\left(B_{2} \dot{z}|\dot{z}|\right) \dot{z} d t
$$

For pure harmonic heaving motion, $z=z_{0} \sin \omega t$, the above equality can be replaced by

$$
z_{0}^{2} \omega B_{e} \int_{0}^{2 \pi} \cos ^{2} \omega t d(\omega t)=4 B_{2} z_{0}^{3} \omega^{2} \int_{0}^{\pi / 2} \cos ^{3} \omega t d(\omega t)
$$




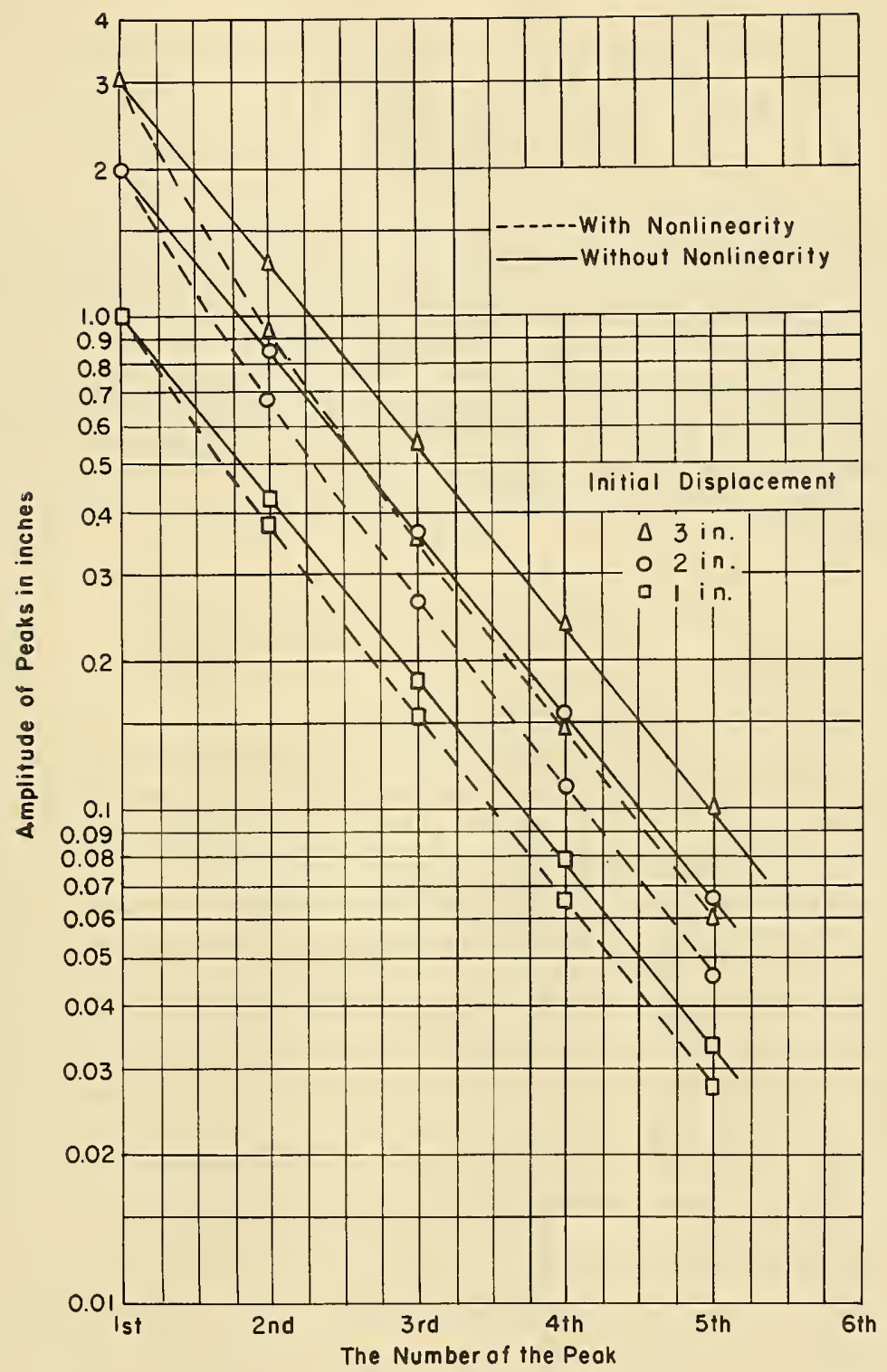

Figure 17 - The Decay of Free Oscillations with and without Nonlinear Damping 
Solving for $B_{e}$, we obtain

$$
B_{e}=4 z_{0} \omega B_{2} \frac{\frac{\sin \omega t \cos ^{2} \omega t}{3}+\left.\frac{2 \sin \omega t}{3}\right|_{0} ^{\pi / 2}}{\left|\frac{\omega t}{2}+\frac{\sin 2 \omega t}{4}\right|_{0}^{2 \pi}}=\frac{8}{3 \pi} z_{0} \omega B_{2}
$$

Jacobsen ${ }^{14}$ has adopted this linearizing procedure for the case of forced steady vibrations with nonlinear damping. The reasoning was intuitive but was substantiated for several cases by comparing the approximate solutions with either experiments or known exact solutions.

Schwesinger ${ }^{15}$ has shown that the linearized equation obtained by equating the work done per cycle yields a "best" one term approximation to the solution; that is, the sum of the forces is minimized rather than being exactly zero.

With this justification, we can replace the equation for the forced nonlinear vibration by a linear one

$$
m \ddot{z}+\left(B_{1}+B_{e}\right) \dot{z}+k z=P_{0} \sin \omega t
$$

where $B_{e}=\frac{8}{3 \pi} \quad z_{0} \omega B_{2}$

In Figure 18 the equivalent damping coefficient $B_{e}$ for 1 -inch and $1 / 2$-inch oscillations is shown. A band is indicated within which all test points fall. The mean line and the limits correspond to the measured values of $B_{2}=0.11 \pm 0.05$. For comparison purposes an average curve for the linear damping coefficient $B_{1}$ is plotted. It appears that $B_{e}$ becomes comparable to $B_{1}$ at higher frequencies and for larger amplitudes of oscillation.

To assess the effect of the nonlinearity on the motion of a ship acted on by a harmonic heaving force, the uncoupled heave equation is considered. Knowing the frequency dependence of the coefficients, the variation of magnification factor with frequency can be computed when the equivalent linear damping term is neglected. This is shown as the solid curve in Figure 19. This frequency dependence can then be recomputed for the nonlinear case by using the mean line in Figure 18 as the equivalent damping term. This cannot be done explicitly since $B_{e}$ depends on the resultant amplitude, so that successive approximations must be used. The resultant solutions depend on the magnitude of the harmonic force imposed on the system and these have been obtained for forces equivalent to static deflections of 1 and 3 inches.

It. should be noted that the curves in Figure 19 are not the usual magnification factor plots although they superficially resemble them. Usually the variation with frequency is shown with the ratio of the damping to critical damping held fixed. In Figure 19, however, as the frequency varies the virtual mass as well as the damping ratio is changing. 


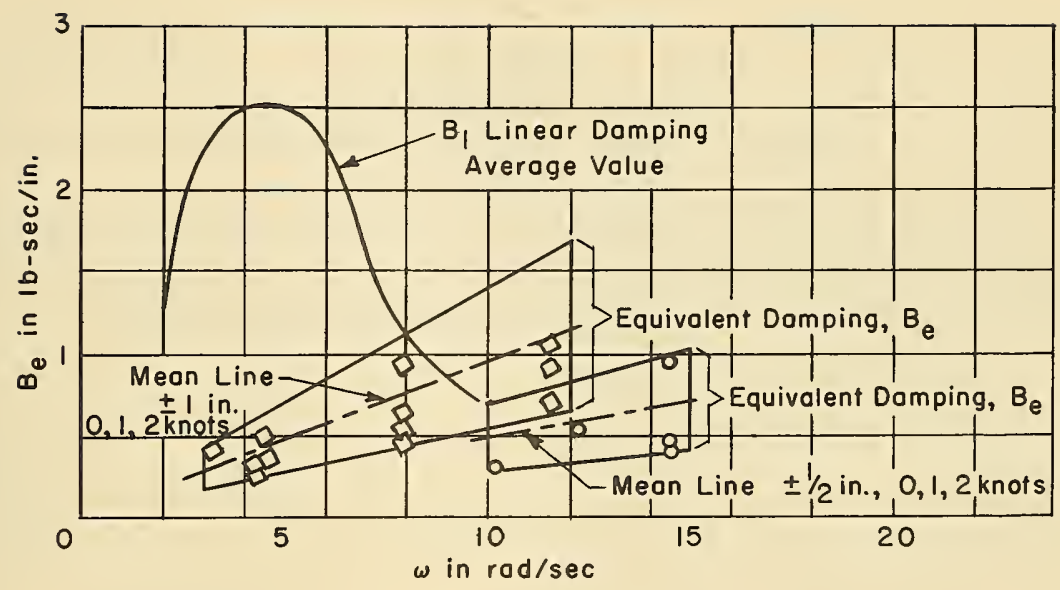

Figure 18 - The Equivalent Damping Coefficient, $B_{e}$

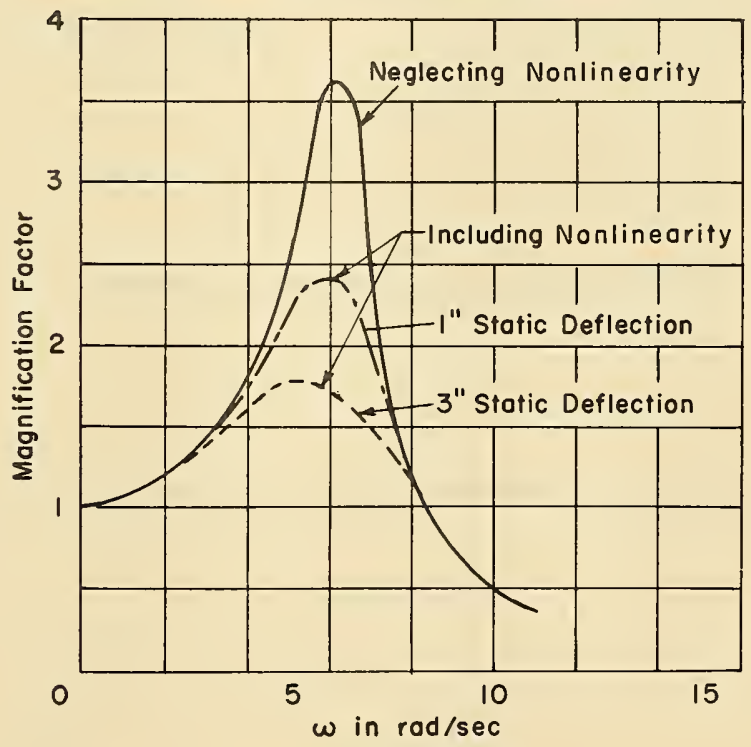

Figure 19 - The Effect of Nonlinear Damping on Forced Vibrations 
It can be seen that at very low and very high frequencies the nonlinearities can be neglected. At low frequencies this is due to the smallness of $B_{e}$, while at high frequencies the magnification factor is insensitive to large changes in damping. However, in the vicinity of resonance, the neglect of the nonlinear damping could result in a serious over-estimate of the motions particularly if the amplitudes of heaving motion are large.

Thus it is apparent that the neglect of nonlinear features of the equations of motion may lead to erroneous motion predictions. Furthermore, this is far from the complete picture since pitching experiments may indicate additional significant nonlinear elements. 


\section{APPENDIX A \\ DYNAMIC CORRECTIONS TO MEASURED FORCES}

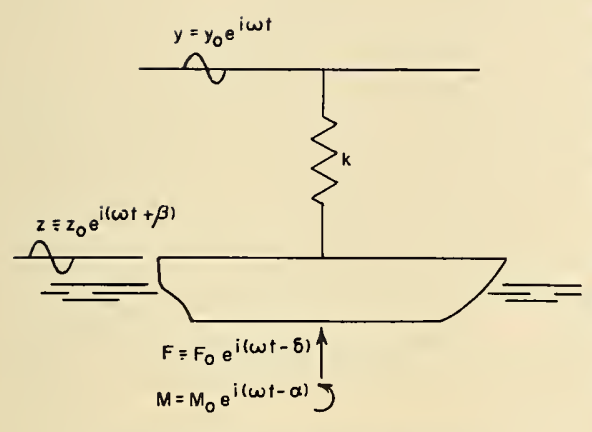

Figure 20 - The Model-Balance Dynamic System

The determination of the coefficients in the equations of motion requires that the displacement of the model, $z(t)$, be known. Unfortunately it is not possible to measure the model motion directly but rather the motion above the balance, $y(t)$. Ideally, with an infinitely stiff balance, the two would be the same. Actually, the balance must be considered to be a spring with high spring constant $k$, for, being a strain gage balance, it must deflect in order to sense the forces.

We wish to know $F(t) / z(t)$ [or $M(t) / z(t)]$ but can only measure $F(t) / y(t)$. Using the complex representations shown in Figure 20

$$
\frac{F(t)}{z(t)}=\frac{F_{0}}{z_{0}} e^{-i(\beta+\delta)}=\frac{F(t)}{y(t)} \times \frac{y(t)}{z(t)}=\frac{F_{0}}{y_{0}} e^{-i \delta} \times \frac{y_{0}}{z_{0}} e^{-i \beta}
$$

Thus $\frac{y_{0}}{z_{0}} e^{-i \beta}$ is a correction which must be applied to the measured quantity

$$
\frac{F_{0}}{y_{0}} e^{-i \delta} \quad \text { or } \frac{M_{0}}{y_{0}} e^{-i}
$$

Assuming as before that the force on the model is proportional to $z, \dot{z}$, and $\ddot{z}$, we can write the force quation

$$
A \ddot{z}+B \dot{z}+C z=k(y-z)
$$

or

$$
A \ddot{z}+B \dot{z}+(C+k) z=k y
$$

substituting $z=z_{0} e^{i(\omega t+\beta)}$ and $y=y_{0} e^{i \omega t}$ we obtain

$$
\left[\left(-A \omega^{2}+C+k\right)+i B \omega\right] z_{0} e^{i \beta}=k y_{0}
$$


or the correction factor is

$$
\frac{y_{0}}{z_{0}} e^{-i \beta}=\frac{-A \omega^{2}+C+k}{k}+i \frac{B \omega}{k}
$$

This equation can only be solved by successive approximation since the added mass (contained within A) and the damping coefficient $B$ are unknown. The procedure would be to solve for $A$ and $B$ assuming $y / z$ equals unity. Then use these values in Equation [6] and obtain $y_{0} / z_{0} e^{-i \beta}$. This correction will yield new values for $A$ and $B$ and the process can be repeated.

For the present system approximate values are $k=5000 \mathrm{lb} / \mathrm{in}$, , and $C=52 \mathrm{lb} / \mathrm{in}$. The value $\beta$ is essentially proportional to frequency and is very small. At $\omega=20 \mathrm{rad} / \mathrm{sec}$, it equals $1 / 3 \mathrm{deg}$. Thus the correction to the measured lift or moment can be considered a real number whose variation with frequency is shown in Figure 21.

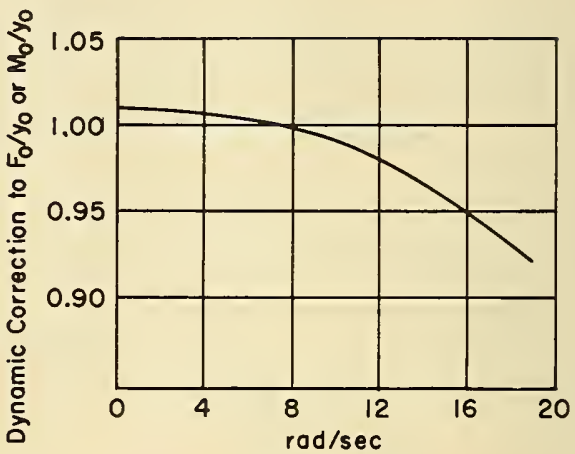

Figure 21 - Dynamic Correction to Measured Forces and Moments 


\section{REFERENCES}

1. Weinblum, G., "Systematische Entwicklung Von Schiffsform," Jahrbuch der Schiffbautechnischen Gesellschaft, Vol. 47 (1953).

2. Den Hartog, J.P., "Mechanical Vibrations," McGraw-Hill (1947).

3. Ursell, F., “Water Waves Generated by Oscillating Bodies," Quarterly Journal Mechanics and Applied Mathematics, Vol. VII, Pt. 4 (1954).

4. Grim, O., "Berechnung Der Durch Schwingungen Eines Schiffskörpers Erzeugten Hydrodynamischen Kräfte," Jahrbuch der Schiffbautechnischen Gesellschaft, Vol. 47 (1953).

5. Prohaska, C.W., "Vibrations Verticales Du Navire," Bulletin Association Technique Maritime and Aeronautique, Vol. 46 (1947).

6. Lewis, F.M., "The Inertia of the Water Surrounding a Vibrating Ship," Transactions Society of Naval Architects and Marine Engineers (1929).

7. Haskind, M.D. ,and Riman, I.S., "A Method of Determining the Pitching and Heaving Characteristics of Ships," David Taylor Model Basin Translation 253 (1955).

8. Havelock, T.Il., "The Coupling of Heave and Pitch Due to Speed of Advance," Transactions Institute of Naval Architects, Vol. 97 (1955).

9. Haskind, M.D., "Oscillation of a Ship in a Calm Sea," Translation in Bulletin No. 1-2, Society of Naval Architects and Marine Engineers (1953).

10. Korvin-Kroukovsky, V.V., "Investigation of Ship Motions in Regular Waves," Transactions Society of Naval Architects and Marine Engineers (1955).

11. Brard, R., "Introduction A L'Étude Théorique Du Tangage En Marche," Bulletin Association Technique Maritime and Aeronautique, Vol. 47, p 456 (1948).

12. Minorsky, N., "Introduction to Non-Linear Mechanics," J.W. Edwards (1947).

13. Kryloff, N. and Bogoliuboff, N., "Introduction to Non-Linear Mechanics," Translation by S. Lefschetz, Princeton University Press (1943).

14. Jacobsen, L.S., "Steady Forced Vibration as Influenced by Damping," Transactions American Society of Mechanical Engineers (1930).

15. Schwesinger, G., "On One-Term Approximations of Forced Non-Harmonic Vibrations," Transactions American Society of Mechanical Engineers (1949). 
INITIAL DISTRIBUTION

Copies

16 CHBUSHIPS, Tech Library (Code 312)

5 Tech Library

1 Deputy and Asst to Chiel (Code 101)

1 Tech Asst to Chiel (Code 106)

1 Apgl Science (Code 370)

1 Ship Design (Code 410)

2 Prel Design Br (Code 420)

1 Prel Design Section (Code 421)

1 Model Basin Liaison (Code 422)

1 Submarines (Code 525)

1 Minesweeping (Code 531)

1 Torpedo Countermeasures (Code 531 M)

4 CHBUDRD, Underwater Ord

2 Code ReU-1

2. Code Re3

2 CHBUAER, Aero and Hydro $\mathrm{Br}(\mathrm{Ad}-3)$

6 CHONR

3 Mech Br (Code 438)

1 Undersea Warfare Br (Code 466)

1 Math Br (Code 432)

1 Naval Sciences Div (Code 460)

1 CO, DNR, New York, N.Y.

1 CD, ONR, Pasadena 1, Calif.

1 CD, ONR, Chicago 1, III.

1 CO, ONR, Boston 10, Mass.

1 CD, ONR, Navy No. 100. FPD Box 39, Landon, Englanó

1 NAVSHIPYD, NORVA

1 NAVSHIPYD, BSN

1 NAVSHIPYD, PTSMH

1 NAVSHIPYD, PUG

1 CO, SURASDEVDET, Key West, Fla.

2 COR, USNDL

I Dr. A. May

1 DIR, USNRL

3 DIR, Langley Aero Lab, Langley Field Va.

1 Dr. C. Kaplan

1 Mr. F.L. Thompson, DIR of Res

2 COR, USN Air Missile Ctr, Point Mugu, Calif. 1 Dr. H.A. Wagner

2 CDR, USNDTS, Underwater Ord Div, Pasadena, Calif.

2 CDR, USNOTS, China Lake, Calif. 1 Dr. E.D. Cooper

1 -CD, USN Air Tech Tiaining Unit,

$$
\text { NAS, Lakehurst, N.J. }
$$

1 CD, USNUDS, New port, R.I.

$1 C D$, Frankford Arsenal oflice of Air Res, Appl Mech Gr, Wright-Patterson AFB, 0 .

2 DIR, Natl BuStand

1 DI. G.H. Keulegan

1 BAR, Bendix Aviation Corp, Teterboro, N.J.

1 ASTIA Ref Ctr, Library of Congress

1 Asst Secy of Defense (Res and Engin) ropies

1 DIR, Dak Ridge Nal Lab, P.D. Bcx P Dak Ridge, Tenn.

1 DIR, Appl Physics Div, Sandia Lab, Albuquerque, N.M.

1 DIR, Ames Aero Lab, Mcfiett Field, Calif.

1 DIR, Lewis Flight Propulsion Lab, Cleveland, Dhio

1 DIR, Alden Hydraulic Lab, Norcester Polytech Inst, Worcester 2, Mass.

2 DIR, Applied Physics Lab, Johns Hepkins Univ, Silver Springs, Md.

1 DIR, Guggenherm Aero Lab, CIT, Pasadena, Calif.

DIR, Fluid Mech Lab, Columbia Univ, New York, N.Y.

1 DIR, Fluid Mech Lab, Univ of Calif, Berkeley, Calif.

3 DIR, ETT, SIT, Hoboken, N.J.

1 Mi. Peters

1 Dr. B.V. Korvin-Kroukovsky

2 DIR, of Aero Res, Natl Advisory Com for Aero, 1512 H St, N.W., Wash 25, D.C

1 DIR, Hydro Lab, Dept of Civil and Sanitary Engin, MIT, Cambridge 39 , Mass.

1 DIR, Exptl Nay Tank, Univ of Mich, Ann Arbor Mich.

1 DIR, Inst for Fluid Dynamics and Appl Wath, Univ of Maryland, College Park, Md.

1 DIR, Inst of Aero Sciences, 2 East 64th St, New York 21, N.Y.

1 DIR, Hydraulics Lab, Uniy of Colorado, Boulder, Colo.

1 DIR, Hydraulic Res Lab, Univ of Conn, Box U-37, Slorrs, Conn.

1 DIR, Scripps Inst of Dceanography, Univ of Calif, La Jolla, Calif.

1 DIR, Dept of Civil Engin, Missouri School of Mines and Netallurgy, Rolla, Mo.

1 DIR, Fluid Mech Lab, New York Univ, New York 53, N.Y.

DIR, Robinson Hydraulic Lab, Dhio State Univ, Columbus, Ohio

1 DiR, Hydraulics Lab, Penn State Univ, University Park, $\mathrm{Pa}$.

1 DIR, Wcods Hole Dceanographic inst, Woods Hole, Mass.

1 DIR, Hydraulic Lab, Univ of Wisconsin, Madison 6 , Wis.

1 DIR, Hydraulics Lab, Univ of Washington, Seattle 5, wash.

1 DIR, ORL, Penn State Univ, University Park, $\mathrm{Pa}$.

1 Admin, Webb Inst of Mav Arch, Glen Cove, Long Island, N.Y.

1 DIR, lowa inst of Hydraulic Res, State Univ of lowa, lawa City, lowa
Copies

1 DIR, St. Antheny Falls Hydraulic Lab, Univ ot Minne solo, Minneapolis 14, Minn.

1 DIR of Res, The Tech Inst, Northwestern Univ, Evanston, III.

1 Head, Dept of Nav Arch and Marine Engin, MIT, Cambridge 39 , Mass.

1 Head, Aero Engin Dept, Catholic Univ, Wash, D.C.

1 Head, Dept of Aero Engin, Johns Hopkins Univ, Baltimore 18, Md.

1 Head, Dept of Aero Engın, Penn State Univ, University Park, $\mathrm{Pa}$

1 Head, Dept of Aero Engın and Appl Mech, PIB, Brooklyn, N.Y.

1 Head, Tech Ret Sec, US Dept of the Interior, Bu of Reclamation, Denver Federal Center, Denver, Colo.

1 Editor, Bibliography of Tech Reperts, Ditice of Tech Services, US Dept ol Commerce, Wash 25, D.C.

1 Editor, Tech Data Digest, Armed Services Tech Inio Agency, Document Service Ctr, Knott Bldg, Dayton 2; Dhio

1 Editor, Engin Index, New York 18, N.Y.

1 Editor, Aero Engin Review, New York 21, N.Y.

2 Editor, Appl Mech Reviews, c/o Southwest Res Inst 8500 Culebra Rd, San Antonio, Texas

1 Hydro Lab, Attn: Exec Com, ClT, Pasadena, Calif.

1 New York Univ inst for Math and Sciences, 25 waverly Place, New York 3, N.Y.

1 SUPSHIPINSORD, Quincy 69, Mass.

2 Newport News Shipbldg and Dry Dock Co, Newport News, Va.

1 Asst Nav Arch

1 DIR Hydraulic Lab

2 SUPSHIPINSORD, New York Shipbldg Corp, Canden, N.J. 1 Mr. J.W. Thempson, Nav Arch (Design)

1 James Forrestal Res Ctr, Princeton Univ, Princeton, M.J., Attn: Mr. Maurice H. Smith Asst to DIR

1 Cornell Aero Lab, Inc. Attn: Librarıan

1 Reed Res, Inc, Wash 7, D.C., Attn: Libratian

1 Shell Devel Co, Houston 2S, Texas, Attn: Librarian

1 Insti Devel Lab, Inc, Needham Hgis 94, Mass. Attn: Mr. C.A. Logan, Engr

1 Dept of Civil Engin, Colorade A and M College, Fort Collins, Colo.

1 Goodyear Aircratt Corp, Akion 15, Dhio

2. Harvard Univ, School of Engin, Cambridge, Mass. 1 G.F. Carrier, Div of Appl Sciences $1 \mathrm{H}$. Emmons

1 Librarian, Amer Soc of Mech Engin, New York 18, N.Y. 
Librarian, Franklin Inst, Parkway at 20th \$t, Philadelphia, $\mathrm{Pa}$.

1 Librarian, Mech Res Library, Illinois Inst of Tech, Tech Ctr, Chicago 16, III.

1 Librarian, Pacific Aero Library, 7660 Beverly Bird, Los Angeles 36, Calif.

Library, Rensselaer Polytechnic Inst, Troy, N.Y.

1 Library, Carnegie Inst of Tech, Pittsburgh 13, $\mathrm{Pa}$.

1 Tech Library, Consolidated Vultee Aircraft Corp, San Diego 12; Calif.

1 Tech Library, The Martin Co, Baltimare 3, Md.

1 Tech Library, Grumann Aircraft Engin Corp, Bethpage, Long Island, N.Y.

1 Tech Library, Lockheed Aircraft Corp. 2555 North Hollywood Way, Burbank, Calif.

1 Tech Libràry, McDonnell Aircraft Corp. Box 516, St. Lotis 3, Mo.

1 Tech Librasy, North American Aviation, Inc, 12241 Lakewood Blvd, Downey, Calit.

1 Tech Library, North American Aviation Inc, El Segundo, Catif.

1 Tech Library, Northrep Aircraft Co 1017 E Broadway, Hawthome, Calif.

1 Tech Library, Pratt and Whitney Aircraft Div, United Aircraft Corp, East Hartford 8, Conn.

1 Chairman, GDAM, Brown Univ, Providence, R.I.

2 Chairman, Dept of Aero Engin, New York Univ. New York 53, N.Y. 1 Consolidated Engin Wind Tunnel

1 Dr. V.L. Streeter, Prot of Hydraulics, Dept of Civil Engr, Univ of Michigan, Ann Arbor, Mich.

1 Prof. G. Birkhoff, DIR, Dept of Malh, Harvard Univ, Cambridge, Mass.

2 Dr. J.V. Wehausen, Exec Editor, Math Reviews, 80 waterman St, Providence 6, R.I.

1 Dr. David Gilbarg, Dept of Math, Indiana Univ, Bloamington, Ind.

1 Prof. K.E. Schoenherr, Dean, College of Engin, Univ of Notre Dame, Notre Dame, Ind.

1 Mr. Maurice L. Anthony, 504 Fitch Road, Chicago Hgts, III.

1 Dr. N.M. Newmark, College of Engin, Univ of Illinois, 207 Talbot Lab, Urbana, III.

1 Dr. C.C. Lin, Dept of Math, MIIT, Cambridge 39 , Mass.

1 Dr. Bruce 6 . Johnston Prof, of Structural Engin, 301 W. Engin Bldg, Univ of Mich, Ann Arbor, Mich.

1 Prof Lydik S. Jacobsen, Dept of Mech Engin Stanford Univ, Berkeley, Calif.

1 Mr. H. Ziebolz, Vice-President, Askania Regulator C0, $240 \mathrm{E}$. Ontario Si, Chicago 11, III.
1 Tech DIR, Ship Structural Com, Natl Res Council, Wash 26, D.C.

2 Dr. M.L. Albertson, Head of Fluid Mech Res, Dept of Civil Engr, Colorado A\&M College Fort Collins, Colo.

1 Prof. A. Yih

Prof M.A. Abkowitz, มIT, Cambridge 39, Mass.

1 Mr. J.P. Breslin, ETT, SIT, 711 Hudson St. Hoboken, N.J.

1 Prof. R.C. Binder, Dept of Mech Engin, Purdue Univ, Lalayette, Ind.

1 Prof N.W. Conner, School of Engin, North Carotina State College, Raleigh, N.C.

1 Dr. F.H. Clauser, Chairman, Dept of Aero, Johns Hopkins Univ, Baltimore 18, Md.

1 VAD'A E.L. Cochtane, USN (Ret) Member, Panel on Submarine Studies, MIT, Cambridge, Mass.

1 CAPT W.S. Diehl, USN, Associate Member, Panel on Subrarine Studies 4501 Lowell St, N.K., Wash, D.C.

1 Mr. Hollinshead de Luce, Central Tech Div, Shipbldg Div, c/o Bethlehem Steel Co, Quincy 69, Mass.

1 Dr. Th. von Karman, 1051 South Marengo St, Pasadena, Calif.

1 Dr. J.H. McMillen, Natl Science Foundation, Wash, O.C.

1 Prof J.H. Miles, Univ of Calif, Los Angeles 24, Calif.

1 Dr. George C. Manning, Prof Nav Arch, MIT, Cambrige 39 , Mass.

1 RADM A.I. McKee, USN, (Ret) Chairman Panel on Submarine Studies and Asst General Manager, Electric Boat Div, General Dyhamics Corp, Groton, Conn.

1 Mr. J.B. Parkinson, Chief, Hydro Div, Langley Ae ro Lab, Langley Field, Va.

1 Dr. W. Pell, Dept of Math, Univ of Kentucky, Louisville, Ky.

1 Dr. M.S. Plesset, Hydro Lab, CIT, Pasadena 4, Calit.

1 Dr. J.M. Robertson, c/O ORL Penn State Univ, University Park, Pa.

1 Prof A. Weinstein, Dept of Wath, Univ of Maryland, College Park, Md.

1 Prof L.1. Schiff, Dept of Physics, Stanford Univ, Berkeley, Calıf.

1 Dr. George Wislicenus, DRL, Johas Hopkins Univ, Baltimore 18 , ud.

1 Prof W. Sears, Graduate School of Engin, Cornell Univ, Ithaca, N.Y.

1 Dr. Stanley Corrsin, Degt of Aero, Johns Hopkins Univ, Baltimore 18, Md.

Dr. C.A. Truesdell, Dept of Math, Univ of Indiana, Bloomington, Ind.

1 Dr. S. Goldstein, Div of Engin and Appl Physics, Harvard Unıv, Cambridge 38 , Mass.
Copies

1 Or. E.V. Laitone, Univ of Calif Berkeley 4, Calif.

1 Dr. L. Trilling, MIT, Cambridge 39, Mass.

1 Prol. F.H. Lewis, Dept of NAME, MIT, Carabridge 39 , Mass.

1 Mr. M.A. Hall, Uniy of Minnesota, Minneapolis 14, Minn.

1 Dr. A. Kantrowitz, Cornell Uniy, Ithaca, N.Y.

1 Dr. R.T. Knapp, Hydro Lab, CIT, Pasadena 4, Calif.

1 Australian Scientific Liaison Dffice, $1800 \mathrm{~K} \mathrm{St}$, N.W., Wash, D.C.

8 ALUSNA, London, England

1 Dir, Hydro Lab, Nall Res Council, Ottawa 2, Canada

1 Prof T.H. Havelock, 8 Westfield Dr. Gosforth, Newcastle-on-Tyne 3 England

1 Mr. C. Wigiey, 6-9 Charterhouse Square, London EC-1 England

1 Dr. Gearg Keinblum, Universitaet Hamburg, Berliner Tor 21, Germany

1 RADM R. Brard, Directeur, Bassin d'Essais des Carenes, 6 Boulevard Victor, Paris (15e), France

1 Dr. L. Malavard, Office National d'Etudes et de Recherches Aeronautiques, $25-39$ Avenue de la Division-LeClerc, Chatillon Sous - Bagneux (Seine) LC, Paris France

1 Gen. Ing. U. Pugliese, Presidenza, Istituto Nazionale per Studi ed Experienze di Architettura Navale, Via della Vasca Navale 89, Roma-Sede, Italy

1 Senor Manuel Lopez - Acevedo, Director, Canat de Experiencias Hidrodinamicas, El Pardo, (Madrid) Carretera de la Sierra, Spain

1 Dr. J. Dieudonne, Directeur, Insitut de Recherches de la Construction Navale, 1 Boulevard Haussmann, Paris ( $9 \mathrm{e})$, France

1 Dir, Nederlandsh Scheepsbouwkundig Proefstation Haagsteeg 2, Wageningen, The Netherlands

1 Prof J.K. Lunde, Skipsmodelltanken, Tyholt, Trondheim, Norway

1 Dr. Hans Edstrand, Dir, Statens Skeppsprouningsanstalt, Goteborg C. 14, Gibrntargalan, Sweden

1 Dir, British Shipbldg Res Assoc, 5 Chesterfield Gardens, Curzon St, London W.1, England

1 Dr. J.F. Allan, Supt, Ship Div, National Physical Lab, Teddington, Middlesex, England

1 Dr. J. Okabe Res Inst for Appl Mech, Kyushu Univ, Hakozaki-Machi, Fukuoka-shi, Japan

1 Prof. J.L. Synge, School of Theoretical Physics, National Univ of Ireland, Dublin, Ireland

\section{Sir R.V. Southwell, 9 Lathburg Rd, Oxford England}

1 Australian Council for Aero, Box 4331 G.P.D. Melbourne, Australia 
1 Chief Supt., Naval Res Establishment c/o Fleet "Aail Office, Halifax, Nova Scotia, Canada

1 Admuralty Res Lab, Teddington, Middlesex, England

1 Arament Res Establishment, near Sevenoaks, Kent, England

1 Dr. J. Ackeret, Institut fur Aerodynamık der Eidgenossiche, Technische Hochschule, Zurich, Switzerland

1 Prof. G.K. Batchelor, Trinity College, Cambrudge Univ, Cambridge, England

1 Prot. L. Escande, Ingenieur, Directeur Ecole Nationale Superieure d'Electrotechnique et d'Hydraulique, 4, Boulevard Qıcuet, Toulouse, France

1 Prof. L. Howarth, Dept of Math, Univ of Bristol, Bristol, England

1 Prof. Harold Jeffreys, St. John's College, Cambridge, England

1 Ir. J. Gerritsma, Delft Shıpbldg Lab, Prof. Mekelweg, Delft, The Netherlands

1 Supt, Aero Div, Natl Physical Lab, Teddington, Middlesex, England

1 DIR, Royal Aircratt Establishment Aero Dept, Farnborough, Hants, England

1 DIR, Aero Res Inst of Sweden. Ranhammarsvagen 12; Ulsvunda, Sweden

1 The Univ of Liverpool, Math Inst, Dept of Appl Math, Grace Library, Liverpool, England

1 Editor, Bulletin of the Britısh Hydro Res Assoc, Netteswell Road, Harlow, Essex, England

1 Dr. Siegfried Schuster, Head, Berlın Madel Basi Versuchsanstalt fur Wasserbau und Schiffbau, Schleuseninsel im Tiergarten, Berlin 87, Germany

1 Dr. H.W. Lerbs, DIR, Hamburg Model Basin, Hamburgische Schiffbau-Versuchsanstalt, Bramfelder Str. 164, Hamburg 33, Germany 





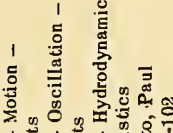

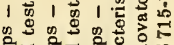

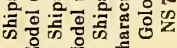

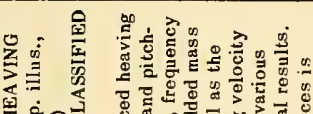

包过

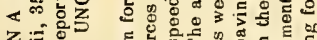

z $=$ :

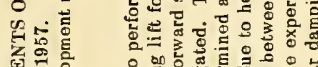

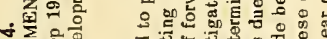

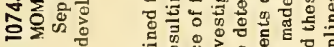

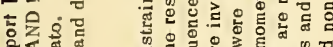

कर क क

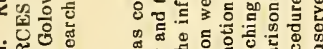

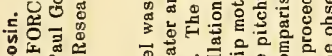

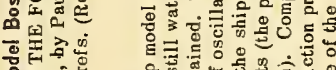

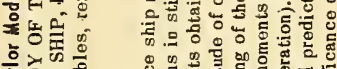

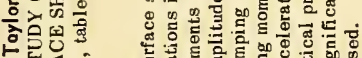

届选

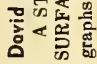

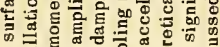

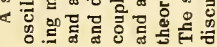

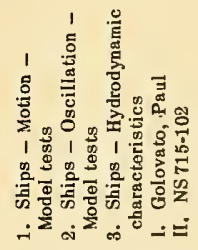

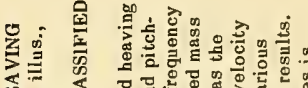

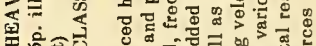

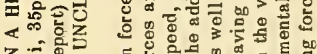

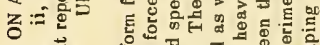

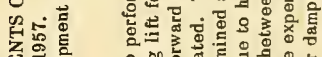

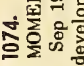

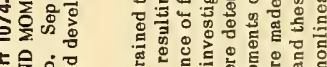

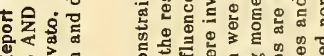

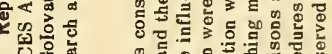

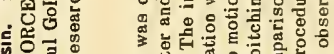

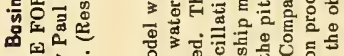

홀

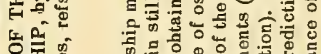

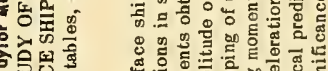

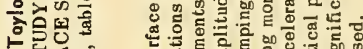

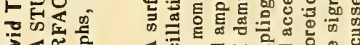

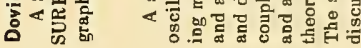

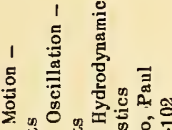

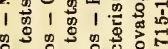

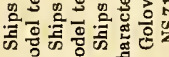

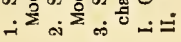

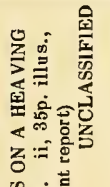

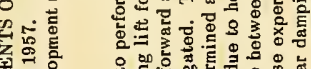

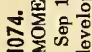
\&

눌

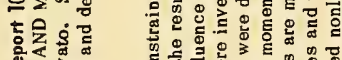

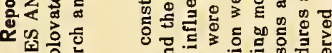

.

ํㅡㅁํㅛ

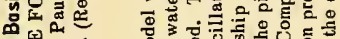

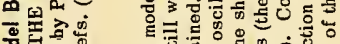

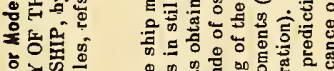

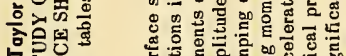

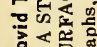

吕《总哭

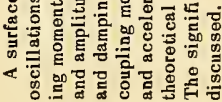

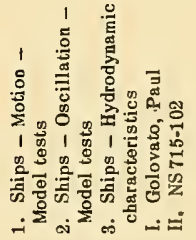

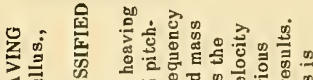

䛼周㝴

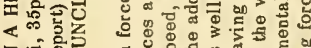

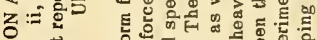

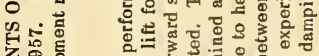

Z实言

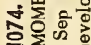

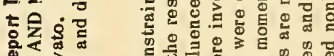

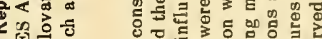

웡영

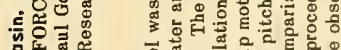

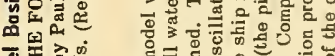

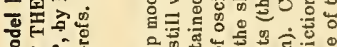

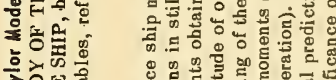

증의

에

号骂

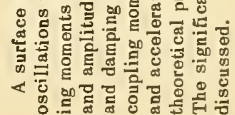





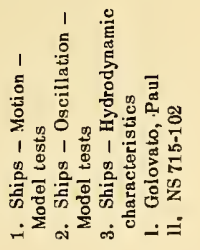

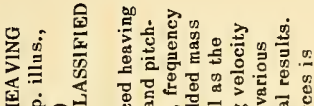

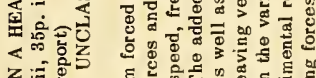

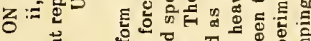

党心

$\sum_{\mathrm{t}}^{20} \mathrm{2}$

额高

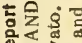

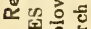

产运范

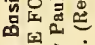

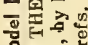

댕

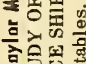

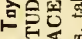

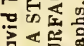

宫《它总

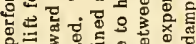

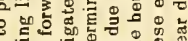
?0

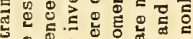
का 8. of

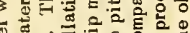

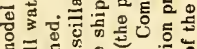
政

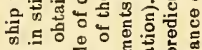

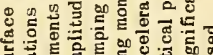

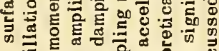

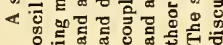

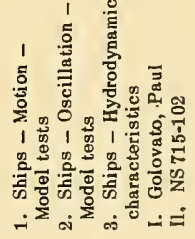

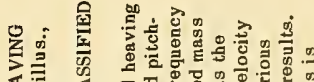

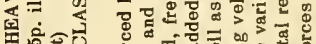

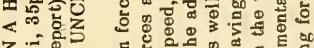

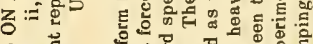

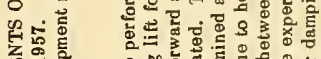
䍃完言

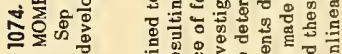

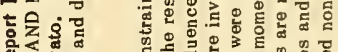

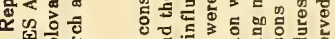
勿定岕 总

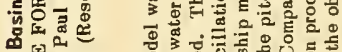

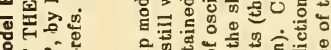

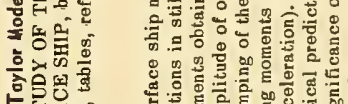

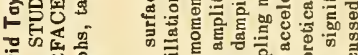

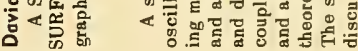

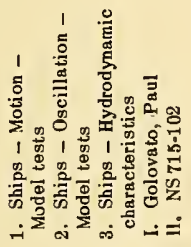

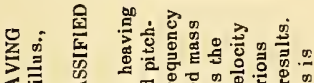

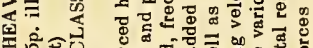

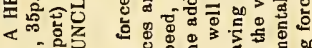

Z : $:$ 足 E

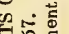

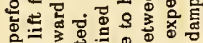

点空

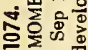

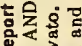

논응

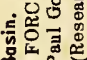

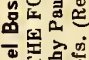

क्षे

형

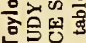

는

究《彭

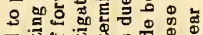

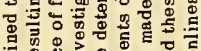

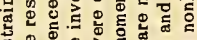

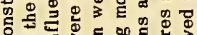

क 记

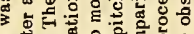

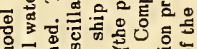

을.

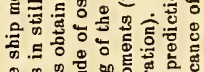

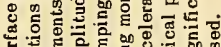

过氙

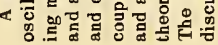

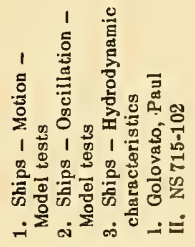

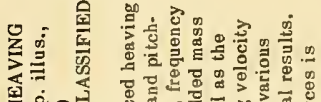

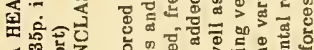

乙:

te

乙年

등ㅎㅀ

言会察焉

농응 흔

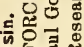

品它寻导

氖实焉

동 夏

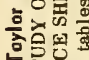

可的密

宫《路

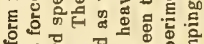

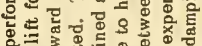

8 吅

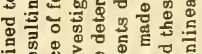

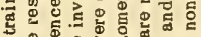

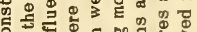

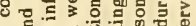

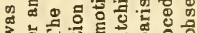

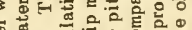

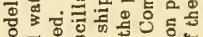

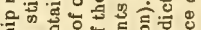

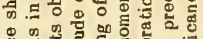

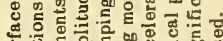

券

×
코용 


